\title{
WEAKLY NONLINEAR-DISSIPATIVE APPROXIMATIONS OF HYPERBOLIC-PARABOLIC SYSTEMS WITH ENTROPY
}

\author{
NING JIANG AND C. DAVID LEVERMORE
}

\begin{abstract}
Hyperbolic-parabolic systems have spatially homogenous stationary states. When the dissipation is weak, one can derive weakly nonlinear-dissipative approximations that govern perturbations of these constant states. These approximations are quadratically nonlinear. When the original system has an entropy, the approximation is formally dissipative in a natural Hilbert space. We show that when the approximation is strictly dissipative it has global weak solutions for all initial data in that Hilbert space. We also prove a weak-strong uniqueness theorem for it. In addition, we give a Kawashima type criterion for this approximation to be strictly dissipative. We apply the theory to the compressible Navier-Stokes system.
\end{abstract}

\section{INTRODUCTION}

We consider hyperbolic-parabolic systems over the $2 \pi$-periodic domain $\mathbb{T}^{d}$ that have the form

$$
\partial_{t} U+\nabla_{x} \cdot F(U)=\nabla_{x} \cdot\left[D(U) \cdot \nabla_{x} U\right] .
$$

These have spatially homogenous stationary solutions. When the dissipation is weak, one can derive a weakly nonlinear-dissipative approximation that governs perturbations $\widetilde{U}$ about any constant solution $U_{o}$. These approximations have the form

$$
\partial_{t} \widetilde{U}+\mathcal{A} \widetilde{U}+\overline{\mathcal{Q}}(\widetilde{U}, \widetilde{U})=\overline{\mathcal{D}} \widetilde{U}
$$

where $\mathcal{A}=F_{U}\left(U_{o}\right) \cdot \nabla_{x}$ is the linearization of the convection operator about $U_{o}$, while quadratic operator $\overline{\mathcal{Q}}$ and the linear operator $\overline{\mathcal{D}}$ are formally given by

$$
\begin{aligned}
\overline{\mathcal{Q}}(Y, Y) & =\lim _{T \rightarrow \infty} \frac{1}{2 T} \int_{-T}^{T} e^{t \mathcal{A}} \nabla_{x} \cdot\left[\frac{1}{2} F_{U U}\left(U_{o}\right)\left(e^{-t \mathcal{A}} Y, e^{-t \mathcal{A}} Y\right)\right] \mathrm{d} t, \\
\overline{\mathcal{D}} Y & =\lim _{T \rightarrow \infty} \frac{1}{2 T} \int_{-T}^{T} e^{t \mathcal{A}} D\left(U_{o}\right): \nabla_{x}^{2}\left(e^{-t \mathcal{A}} Y\right) \mathrm{d} t .
\end{aligned}
$$

Such weakly nonlinear-dissipative approximations arise when studying incompressible limits of the compressible Navier-Stokes system [8,32], global regularity of fast rotating Navier-Stokes and Euler equations [1, 2, 3], asymptotic limits in equations of geophysical fluid dynamics $[11,12]$, and fast singular limits of hyperbolic and parabolic PDE's [35, 14, 15].

We show that if the original system (1.1) has a thrice differentiable convex entropy structure then the approximating system (1.2) is formally dissipative in the Hilbert space $\mathbb{H}$ whose inner product is given by

$$
(\widetilde{U} \mid \widetilde{V})_{\mathbb{H}}=\frac{1}{(2 \pi)^{d}} \int_{\mathbb{T}^{d}} H_{U U}\left(U_{o}\right)(\widetilde{U}(x), \widetilde{V}(x)) \mathrm{d} x
$$

Date: April 16, 2009. 
where $H_{U U}\left(U_{o}\right)$ is the Hessian of the strictly convex entropy density $H(U)$ at $U_{o}$. This dissipation property follows because the entropy structure implies that $\mathcal{A}$ is skew-adjoint in $\mathbb{H}$, that $\overline{\mathcal{D}}$ is nonpositive definite in $\mathbb{H}$, and that $\overline{\mathcal{Q}}$ formally satisfies the cyclic identity

$$
0=(\widetilde{U} \mid \overline{\mathcal{Q}}(\widetilde{V}, \widetilde{W}))_{\mathbb{H}}+(\widetilde{V} \mid \overline{\mathcal{Q}}(\widetilde{W}, \widetilde{U}))_{\mathbb{H}}+(\widetilde{W} \mid \overline{\mathcal{Q}}(\widetilde{U}, \widetilde{V}))_{\mathbb{H}} .
$$

We show that if $\overline{\mathcal{D}}$ is also strictly dissipative then the approximating system (1.2) has a Leraytype global weak solution for all initial data in $\mathbb{H}$. We cannot establish the uniqueness of these solutions. Indeed, when (1.1) is the Navier-Stokes system of gas dynamics then (1.2) includes the incompressible Navier-Stokes system as a subsystem. The uniqueness question therefore includes the uniqueness question for the incompressible Navier-Stokes system. We can however use the cyclic identity (1.4) to prove a so-called weak-strong uniqueness theorem for the approximating system (1.2).

It will be easily seen that $\overline{\mathcal{D}}$ will be nonnegative definite if and only if the linear operators $\mathcal{A}=F_{U}\left(U_{o}\right) \cdot \nabla_{x}$ and $\mathcal{D}=D\left(U_{o}\right): \nabla_{x}^{2}$ satisfy the Kawashima condition:

no nonconstant eigenfunction of $\mathcal{A}$ is in the null space of $\mathcal{D}$.

In one spatial dimension it is known that the Kawashima condition implies that $\overline{\mathcal{D}}$ is strictly dissipative [25]. We give a stronger Kawashima-type criterion for $\overline{\mathcal{D}}$ to be strictly dissipative in higher dimensions.

Our paper is laid out as follows. Section 2 presents the entropy structure we will impose on system (1.1). Section 3 presents the weakly nonlinear-dissipative approximation (1.2) and its relation to the Kawashima condition (1.5). Section 4 develops the properties of the averaged operators (1.3) that we will need later. This includes a proof of the cyclic identity (1.4). Section 5 presents our Kawashima-type criterion for $\overline{\mathcal{D}}$ to be strictly dissipative. Section 6 contains our existence and weak-strong uniqueness theorems. Finally, section 7 applies the theory to the compressible Navier-Stokes system of gas dynamics.

\section{Hyperbolic-Parabolic Systems with Entropy}

We consider hyperbolic-parabolic systems over $\mathbb{T}^{d}$ in the divergence form

$$
\partial_{t} U+\nabla_{x} \cdot F(U)=\nabla_{x} \cdot\left[D(U) \cdot \nabla_{x} U\right]
$$

where $U(x, t)$ is a density vector over $(x, t) \in \mathbb{T}^{d} \times \mathbb{R}_{+}$that takes values in $\mathcal{U}^{c} \subset \mathbb{R}^{\mathrm{N}}$. Here $\mathbb{T}^{d}=\mathbb{R}^{d} /(2 \pi \mathbb{Z})^{d}$ is the $2 \pi$-periodic torus and $\mathcal{U}^{c}$ is the closure of a convex domain $\mathcal{U} \subset \mathbb{R}^{\mathrm{N}}$. We assume that the flux tensor $F: \mathcal{U} \rightarrow \mathbb{R}^{d \times \mathrm{N}}$ is twice continuously differentiable such that

$$
\partial_{t} U+\nabla_{x} \cdot F(U)=0 \text { is hyperbolic, }
$$

while the diffusion tensor $D: \mathcal{U} \rightarrow \mathbb{R}^{d \times d \times N \times N}$ is continuously differentiable such that

$$
\partial_{t} U=\nabla_{x} \cdot\left[D(U) \cdot \nabla_{x} U\right] \text { is parabolic . }
$$

Recall that system (2.2) is said to be hyperbolic if for every $U \in \mathcal{U}$ and every $\xi \in \mathbb{R}^{d}$ the $N \times N$ matrix $F_{U}(U) \cdot \xi$ is diagonalizable within the reals - i.e. it has a complete set of real eigenvectors. System (2.2) is said to be strictly hyperbolic if moreover the eigenvalues of $F_{U}(U) \cdot \xi$ are distinct. Recall that system (2.3) is said to be parabolic if for every $U \in \mathcal{U}$ and every $\xi \in \mathbb{R}^{d}$ the $N \times N$ matrix $D(U): \xi^{\otimes 2}$ is diagonalizable within the reals and has nonnegative eigenvalues. System (2.3) is said to be strictly parabolic if moreover the eigenvalues of $D(U): \xi^{\otimes 2}$ are positive. Many studies of hyperbolic-parabolic systems assume that system (2.2) is strictly hyperbolic while system (2.3) is strictly parabolic. We will not do that here. Rather, we 
will assume that system (2.1) has a strictly convex entropy and satisfies certain nonsingularity conditions.

2.1. Entropy Structure. We say that $H: \mathcal{U} \rightarrow \mathbb{R}$ is a strictly convex entropy for the system (2.1) when $H$ is twice continuously differentiable over $\mathcal{U}$ and for every $U \in \mathcal{U}$

$$
\begin{array}{ll}
\text { (i) } H_{U U}(U) & \text { is positive definite, } \\
\text { (ii) } H_{U U}(U) F_{U}(U) \cdot \xi & \text { is symmetric for every } \xi \in \mathbb{R}^{d}, \\
\text { (iii) } H_{U U}(U) D(U) & \text { is symmetric and nonnegative definite. }
\end{array}
$$

The existence of such a strictly convex entropy implies that system (2.1) is hyperbolic-parabolic. The compressible Navier-Stokes system is a hyperbolic-parabolic system that is neither strictly hyperbolic nor strictly parabolic, yet has a strictly convex entropy. We will study this example in Section 7. There are many other systems from physics that fit into this framework [5].

Condition (ii) in (2.4) implies there exists $J: \mathcal{U} \rightarrow \mathbb{R}^{d}$ that is twice continuously differentiable such that

$$
H_{U}(U) F_{U}(U)=J_{U}(U) .
$$

It follows that if $U(x, t)$ is a classical solution of $(2.1)$ that takes its values in $\mathcal{U}$ then it satisfies

$$
\partial_{t} H(U)+\nabla_{x} \cdot J(U)=\nabla_{x} \cdot\left[H_{U}(U) D(U) \cdot \nabla_{x} U\right]-\nabla_{x} H_{U}(U) \cdot D(U) \cdot \nabla_{x} U .
$$

Condition (iii) in (2.4) implies that for every differentiable $U: \mathbb{T}^{d} \rightarrow \mathbb{R}^{\mathrm{N}}$ with $U(x) \in \mathcal{U}$

$$
\nabla_{x} H_{U}(U) \cdot D(U) \cdot \nabla_{x} U=\nabla_{x} U^{T} \cdot H_{U U}(U) D(U) \cdot \nabla_{x} U \geq 0 .
$$

We thereby see that (2.6) is a local dissipation law for $H(U)$. When (2.6) is integrated over $\mathbb{T}^{d}$ one obtains the global dissipation law

$$
\begin{aligned}
\frac{\mathrm{d}}{\mathrm{d} t} \int H(U) \mathrm{d} x & =-\int \nabla_{x} H_{U}(U) \cdot D(U) \cdot \nabla_{x} U \mathrm{~d} x \\
& =-\int \nabla_{x} U^{T} \cdot H_{U U}(U) D(U) \cdot \nabla_{x} U \mathrm{~d} x \leq 0 .
\end{aligned}
$$

2.2. Nonsingularity Condition. The class of stationary classical solutions of (2.1) that take values in $\mathcal{U}$ is constrained by the entropy structure. It follows from (2.8) that every such solution satisfies

$$
\int \nabla_{x} U^{T} \cdot H_{U U}(U) D(U) \cdot \nabla_{x} U \mathrm{~d} x=0
$$

which by (2.7) implies that

$$
\nabla_{x} U^{T} \cdot H_{U U}(U) D(U) \cdot \nabla_{x} U=0 .
$$

Conditions (i) and (iii) then imply that $D(U) \cdot \nabla_{x} U=0$, which when plugged into (2.1) with $\partial_{t} U=0$ yields $\nabla_{x} \cdot F(U)=0$. We thereby see that every classical solution of (2.1) that takes its values in $\mathcal{U}$ satisfies

$$
\nabla_{x} \cdot F(U)=0, \quad D(U) \cdot \nabla_{x} U=0 .
$$

In general this is not enough information to conclude that $U$ is a constant. 
We consider hyperbolic-parabolic systems over $\mathbb{T}^{d}$ that are nonsingular in the sense that for every $U \in \mathcal{U}$ and every continuously differentiable $\widetilde{U}: \mathbb{T}^{d} \rightarrow \mathbb{R}^{N}$ one has that

$$
\left.\begin{array}{rl}
F_{U}(U) \cdot \nabla_{x} \widetilde{U} & =0 \\
D(U) \cdot \nabla_{x} \widetilde{U} & =0
\end{array}\right\} \quad \Longrightarrow \quad \nabla_{x} \widetilde{U}=0 .
$$

With this additional assumption we can prove the following.

Lemma 2.1. If the hyperbolic-parabolic system (2.1) has a strictly convex entropy (2.4) and satisfies the nonsingularity condition (2.10) then its only stationary, spatially periodic classical solutions that take their values in $\mathcal{U}$ are constant solutions.

In the next section we will strengthen the nonsingularity condition (2.10).

\section{Weakly Nonlinear-Dissipative Approximations}

We now consider weakly nonlinear-dissipative (WND) approximations to hyperbolic-parabolic systems of the form (2.1). In order to see how these approximations depend on the choice of dependent variables, we express (2.1) in the form

$$
\partial_{t} U(W)+\nabla_{x} \cdot F(U(W))=\nabla_{x} \cdot\left[D(U(W)) \cdot \nabla_{x} U(W)\right] .
$$

Here the mapping $U: \mathcal{W} \rightarrow \mathcal{U}$ is assumed to be a twice continuously differentiable bijection and have a nonsingular Jacobian. This means that its inverse mapping $U^{-1}: \mathcal{U} \rightarrow \mathcal{W}$ will also be a twice continuously differentiable bijection and have a nonsingular Jacobian.

3.1. Linearization and the Kawashima Condition. In order to motivate our approximation, let us first consider the linearization of the hyperbolic-parabolic system (3.1) about some constant state $W_{o} \in \mathcal{W}$. This is

$$
\partial_{t} \widetilde{W}+A_{o} \cdot \nabla_{x} \widetilde{W}=B_{o}: \nabla_{x}^{2} \widetilde{W}
$$

where $A_{o} \in \mathbb{R}^{d \times \mathrm{N} \times \mathrm{N}}$, and $B_{o} \in \mathbb{R}^{d \times d \times \mathrm{N} \times \mathrm{N}}$ are defined by

$$
A_{o}=R_{o}^{-1} F_{U}\left(U_{o}\right) R_{o}, \quad B_{o}=R_{o}^{-1} D\left(U_{o}\right) R_{o},
$$

with $U_{o} \in \mathcal{U}$ and $R_{o} \in \mathbb{R}^{\mathrm{N} \times \mathrm{N}}$ given by

$$
U_{o}=U\left(W_{o}\right), \quad R_{o}=\partial_{V} U\left(W_{o}\right) .
$$

If $H: \mathcal{U} \rightarrow \mathbb{R}$ is a strictly convex entropy for system (2.1) then system (3.2) is symmetrized by the positive definite matrix

$$
G_{o}=R_{o}^{T} H_{U U}\left(U_{o}\right) R_{o} .
$$

Specifically, one sees from (2.4) and (3.3) that

$$
\begin{aligned}
G_{o} A_{o} \cdot \xi & =R_{o}^{T} H_{U U}\left(U_{o}\right) F_{U}\left(U_{o}\right) \cdot \xi R_{o} & & \text { is symmetric for every } \xi \in \mathbb{R}^{d}, \\
G_{o} B_{o} & =R_{o}^{T} H_{U U}\left(U_{o}\right) D\left(U_{o}\right) R_{o} & & \text { is symmetric and nonnegative definite. }
\end{aligned}
$$

The solutions of (3.2) thereby satisfy the local dissipation law

$$
\partial_{t}\left(\frac{1}{2} \widetilde{W}^{T} G_{o} \widetilde{W}\right)+\nabla_{x} \cdot\left(\frac{1}{2} \widetilde{W}^{T} G_{o} A_{o} \widetilde{W}\right)=\nabla_{x} \cdot\left(\widetilde{W^{T}} G_{o} B_{o} \cdot \nabla_{x} \widetilde{W}\right)-\nabla_{x} \widetilde{W}^{T} \cdot G_{o} B_{o} \cdot \nabla_{x} \widetilde{W}
$$

When this equation is integrated over $\mathbb{T}^{d}$ one obtains the global dissipation law

$$
\frac{\mathrm{d}}{\mathrm{d} t} \int \frac{1}{2} \widetilde{W}^{T} G_{o} \widetilde{W} \mathrm{~d} x=-\int \nabla_{x} \widetilde{W}^{T} \cdot G_{o} B_{o} \cdot \nabla_{x} \widetilde{W} \mathrm{~d} x \leq 0
$$


The initial-value problem for system (3.2) is therefore naturally well-posed in the Hilbert space $\mathbb{H}=L^{2}\left(\mathrm{~d} x ; \mathbb{R}^{\mathrm{N}}\right)$ equipped with the inner product

$$
\left(\widetilde{W}_{1} \mid \widetilde{W}_{2}\right)_{\mathbb{H}}=\frac{1}{(2 \pi)^{d}} \int_{\mathbb{T}^{d}} \widetilde{W}_{1}^{T} G_{o} \widetilde{W}_{2} \mathrm{~d} x
$$

where $G_{o}$ is given by (3.5).

By arguing as we did in the nonlinear settings, every stationary, spatially periodic classical solution of system (3.2) must satisfy

$$
A_{o} \cdot \nabla_{x} \widetilde{W}=0, \quad B_{o} \cdot \nabla_{x} \widetilde{W}=0 .
$$

We can conclude that $\nabla_{x} \widetilde{W}=0$ if system (2.1) satisfies the nonsingularity condition (2.10). In that case the only stationary, spatially periodic classical solutions of system (3.2) are the constant solutions.

It is natural to ask if every solution of system (3.2) in $\mathbb{H}$ will decay to a constant solution as $t \rightarrow \infty$. Kawashima [23, 24] gave an elegant characterization of when this is the case in terms of the skew-adjoint operator $\mathcal{A}$ and the self-adjoint operator $\mathcal{D}$ that are formally given by

$$
\mathcal{A}=A_{o} \cdot \nabla_{x}, \quad \mathcal{D}=B_{o}: \nabla_{x}^{2} .
$$

Because $\mathcal{A}=A_{o} \cdot \nabla_{x}$ is skew-adjoint in $\mathbb{H}$, its spectrum, $\operatorname{Sp}(\mathcal{A})$, is purely imaginary. When the spatial domain is bounded $\operatorname{Sp}(\mathcal{A})$ only contains eigenvalues. Moreover, these eigenvalues and their corresponding eigenfunctions are easily computed by Fourier methods when the spatial domain is $\mathbb{T}^{d}$. Kawashima gave the following characterization.

Theorem 3.1. Every solution of system (3.2) in $\mathbb{H}$ will decay to a constant solution as $t \rightarrow \infty$ if and only if

$$
\text { no nonconstant eigenfunction of } \mathcal{A} \text { is in the null space of } \mathcal{D} \text {. }
$$

Remark. The Kawashima condition (3.7) is clearly necessary for every solution of system (3.2) in $\mathbb{H}$ to decay to a constant solution as $t \rightarrow \infty$. Indeed, if $V$ is nonconstant eigenfunction of $\mathcal{A}$ for the eigenvalue $i \omega$ such that $V$ lies in the null space of $\mathcal{D}$ then the real part of $e^{-i \omega t} V$ is a real-valued solution of (3.2) that does not decay to a constant solution as $t \rightarrow \infty$.

Remark. The Kawashima condition (3.7) is stronger than our nonsingular condition (2.10). It has been used to obtain similar results regarding the existence, regularity, and decay as $t \rightarrow \infty$ of global solutions to nonlinear systems [4, 18, 19, 25, 26, 34, 37]. Villani has generalized it in his theory of hypercoercivity [38] where he discusses its relation to the older Hörmander hypoellipticity condition [20].

3.2. Weak Dissipation, Two-Time Asymptotics. It is more interesting to consider regimes in which the dissipation is weak. Introduce the nondimensional (small) parameter $\epsilon>0$ so that

$$
\partial_{t} U\left(W_{\epsilon}\right)+\nabla_{x} \cdot F\left(U\left(W_{\epsilon}\right)\right)=\epsilon \nabla_{x} \cdot\left[D\left(U\left(W_{\epsilon}\right)\right) \cdot \nabla_{x} U\left(W_{\epsilon}\right)\right],
$$

Let $W_{o} \in \mathcal{W}$ and set

$$
W_{\epsilon}=W_{o}+\epsilon \widetilde{W}+\epsilon^{2} \widetilde{W}^{(2)}+O\left(\epsilon^{3}\right) .
$$

Then

$$
U\left(W_{\epsilon}\right)=U_{o}+\epsilon R_{o} \widetilde{W}+\epsilon^{2} R_{o}\left(\widetilde{W}^{(2)}+S_{o}(\widetilde{W}, \widetilde{W})\right)+O\left(\epsilon^{3}\right),
$$

where $U_{o}$ and $R_{o}$ are given by (3.4) while $S_{o}$ is defined by

$$
S_{o}(\widetilde{W}, \widetilde{W})=\frac{1}{2} R_{o}^{-1} \partial_{W W} U\left(W_{o}\right)(\widetilde{W}, \widetilde{W})
$$


To first order in $\epsilon$ we see that $\widetilde{W}$ satisfies

$$
\partial_{t} \widetilde{W}+A_{o} \cdot \nabla_{x} \widetilde{W}=0
$$

The solution of this equation is given by

$$
\widetilde{W}(t)=e^{-t \mathcal{A}} \widetilde{W^{\text {in }}}, \quad \text { where } \mathcal{A}=A_{o} \cdot \nabla_{x} .
$$

Because $\mathcal{A}$ is skew-adjoint on $\mathbb{H}$, the solution operator $e^{-t \mathcal{A}}$ is strongly continuous, one parameter group of unitary operators on $\mathbb{H}$. This approximation cannot be valid uniformly in time because (1) the solutions of (3.9) do not decay as $t \rightarrow \infty$ and (2) there are generally nonconstant stationary solutions of $(3.9)$ when $d \geq 2$.

In order to overcome these problems, one has to introduce a slow time scale $\tau=\epsilon t$ into the asymptotics and consider

$$
\epsilon \partial_{\tau} U\left(W_{\epsilon}\right)+\partial_{t} U\left(W_{\epsilon}\right)+\nabla_{x} \cdot F\left(U\left(W_{\epsilon}\right)\right)=\epsilon \nabla_{x} \cdot\left[D\left(U\left(W_{\epsilon}\right)\right) \cdot \nabla_{x} U\left(W_{\epsilon}\right)\right] .
$$

To first order we see that $\widetilde{W}$ still satisfies (3.8). Hence,

$$
\widetilde{W}(t, \tau)=e^{-t \mathcal{A}} \widetilde{Y}(\tau),
$$

where the $\tau$ dependence of $\tilde{Y}$ has yet to be determined.

To the second order in $\epsilon$ we see that

$$
\partial_{t} \widetilde{W}^{(2)}+\mathcal{A} \widetilde{W}^{(2)}=-\partial_{\tau} \widetilde{W}-\nabla_{x} \cdot Q_{o}(\widetilde{W}, \widetilde{W})+B_{o}: \nabla_{x}^{2} \widetilde{W}-\left(\partial_{t}+\mathcal{A}\right) S_{o}(\widetilde{W}, \widetilde{W}),
$$

where

$$
Q_{o}(\widetilde{W}, \widetilde{W})=\frac{1}{2} R_{o}^{-1} F_{U U}\left(U_{o}\right)\left(R_{o} \widetilde{W}, R_{o} \widetilde{W}\right) .
$$

The right-hand side of (3.11) is an almost periodic function of $t$. For $\widetilde{W}^{(2)}$ to be an almost periodic, we must require

$$
\lim _{T \rightarrow \infty} \frac{1}{2 T} \int_{-T}^{T} e^{t \mathcal{A}}\left[\partial_{\tau} \widetilde{W}+\nabla_{x} \cdot Q_{o}(\widetilde{W}, \widetilde{W})-B_{o}: \nabla_{x}^{2} \widetilde{W}\right] \mathrm{d} t=0 .
$$

Hence, because $\widetilde{W}$ is given by (3.10), we see that $\widetilde{Y}(\tau)$ satisfies

$$
\partial_{\tau} \tilde{Y}+\overline{\mathcal{Q}}(\tilde{Y}, \tilde{Y})=\overline{\mathcal{D}} \tilde{Y}
$$

where the operators $\overline{\mathcal{Q}}$ and $\overline{\mathcal{D}}$ are formally defined by

$$
\begin{aligned}
\overline{\mathcal{Q}}(Y, Y) & =\lim _{T \rightarrow \infty} \frac{1}{2 T} \int_{-T}^{T} e^{t \mathcal{A}} \mathcal{Q}\left(e^{-t \mathcal{A}} Y, e^{-t \mathcal{A}} Y\right) \mathrm{d} t, \\
\overline{\mathcal{D}} Y & =\lim _{T \rightarrow \infty} \frac{1}{2 T} \int_{-T}^{T} e^{t \mathcal{A}} \mathcal{D} e^{-t \mathcal{A}} Y \mathrm{~d} t,
\end{aligned}
$$

with the operators $\mathcal{Q}$ and $\mathcal{D}$ being given by

$$
\mathcal{Q}(W, W)=\nabla_{x} \cdot Q_{o}(W, W), \quad \mathcal{D} W=B_{o}: \nabla_{x}^{2} W .
$$

It is easily checked from formulas (3.14) that

$$
\overline{\mathcal{Q}}\left(e^{-t \mathcal{A}} W, e^{-t \mathcal{A}} W\right)=e^{-t \mathcal{A}} \overline{\mathcal{Q}}(W, W), \quad \overline{\mathcal{D}} e^{-t \mathcal{A}}=e^{-t \mathcal{A}} \overline{\mathcal{D}} .
$$

It then follows from (3.10) and (3.13) that $\widetilde{W}(t, \epsilon t)$ satisfies

$$
\partial_{t} \widetilde{W}+\mathcal{A} \widetilde{W}+\epsilon \overline{\mathcal{Q}}(\widetilde{W}, \widetilde{W})=\epsilon \overline{\mathcal{D}} \widetilde{W}
$$


Setting $\epsilon=1$, we call this the weakly nonlinear-dissipative approximation of the hyperbolicparabolic system (3.1).

3.3. Change of Dependent Variables. Suppose we had expressed the system (3.6) in terms of different dependent variables $W^{\prime}$ :

$$
\partial_{t} U^{\prime}\left(W_{\epsilon}^{\prime}\right)+\nabla_{x} \cdot F\left(U^{\prime}\left(W_{\epsilon}^{\prime}\right)\right)=\epsilon \nabla_{x} \cdot\left[D\left(U^{\prime}\left(W_{\epsilon}^{\prime}\right)\right) \cdot \nabla_{x} U^{\prime}\left(W_{\epsilon}^{\prime}\right)\right]
$$

where $U^{\prime}: \mathcal{W}^{\prime} \rightarrow \mathcal{U}$ is a twice continuously differentiable bijection with a nonsingular Jacobian. Let $W_{o}^{\prime}$ be the unique constant state such that $U^{\prime}\left(W_{o}^{\prime}\right)=U_{o}=U\left(W_{o}\right)$. If we approximate solutions of (3.16) that are near $W_{o}^{\prime}$ just as we approximated solutions of (3.8) that are near $W_{o}$ then to leading order we obtain

$$
\partial_{t} \widetilde{W^{\prime}}+A_{o}^{\prime} \cdot \nabla_{x} \widetilde{W^{\prime}}=0
$$

where $A_{o}^{\prime}=T_{o}^{-1} A_{o} T_{o}$ with

$$
T_{o}=\left.\partial_{W^{\prime}}\left(U^{-1}\left(U^{\prime}\left(W^{\prime}\right)\right)\right)\right|_{W^{\prime}=W_{o}^{\prime}}=R_{o}^{-1} R_{o}^{\prime} .
$$

In other words, the leading order approximation of the transformed system (3.16) is the leading order approximation of the original system transformed by the associated linear change of variables $\widetilde{W}=T_{o} \widetilde{W^{\prime}}$.

Remarkably, the same transformation property holds for the weakly nonlinear-dissipative approximation! Specifically, if the weakly nonlinear-dissipative approximation of the primed system (3.16) is

$$
\partial_{t} \widetilde{W^{\prime}}+\mathcal{A}^{\prime} \widetilde{W^{\prime}}+\epsilon \overline{\mathcal{Q}}^{\prime}\left(\widetilde{W^{\prime}}, \widetilde{W^{\prime}}\right)=\epsilon \overline{\mathcal{D}}^{\prime} \widetilde{W}
$$

then it is related to the weakly nonlinear-dissipative approximation of the unprimed system by

$$
\mathcal{A}^{\prime}=T_{o}^{-1} \mathcal{A} T_{o}, \quad \overline{\mathcal{Q}}^{\prime}\left(\widetilde{W^{\prime}}, \widetilde{W^{\prime}}\right)=T_{o}^{-1} \overline{\mathcal{Q}}\left(T_{o} \widetilde{W}^{\prime}, T_{o} \widetilde{W}^{\prime}\right), \quad \overline{\mathcal{D}}^{\prime}=T_{o}^{-1} \overline{\mathcal{D}} T_{o},
$$

where $T_{o}$ is again given by (3.17). In other words, it is simply the original nonlinear-dissipative approximation transformed by the associated linear change of variables $\widetilde{W}=T_{o} \widetilde{W}^{\prime}$. This fact allows us to derive the weakly nonlinear-dissipative approximation of system (3.8) in any set of dependent variables we choose becasue the result is unique up to the transformation (3.18).

\section{Averaged Operators}

In this section we collect some properties of the averaged operators $\overline{\mathcal{Q}}$ and $\overline{\mathcal{D}}$.

4.1. Spectral Formulas for the Averaged Operators. Because $\mathcal{A}=A_{o} \cdot \nabla_{x}$ is skew-adjoint in $\mathbb{H}$, its spectrum, $\operatorname{Sp}(\mathcal{A})$, is purely imaginary. Define

$$
\sigma(\mathcal{A})=\{\omega \in \mathbb{R}: i \omega \in \operatorname{Sp}(\mathcal{A})\} .
$$

For every $\omega \in \sigma(\mathcal{A})$ let $\mathbb{H}_{\omega}$ denote the eigenspace associated with the eigenvalue $i \omega$ of $\mathcal{A}$ and let $E_{\omega}$ be the $\mathbb{H}$-orthogonal projection onto $\mathbb{H}_{\omega}$. Then for every $\omega \in \sigma(\mathcal{A})$ one has

$$
\begin{gathered}
\left(E_{\omega} W_{1} \mid W_{2}\right)_{\mathbb{H}}=\left(W_{1} \mid E_{\omega} W_{2}\right)_{\mathbb{H}} \quad \text { for every } W_{1}, W_{2} \in \mathbb{H}, \\
E_{\omega}^{2}=E_{\omega}, \quad \mathbb{H}_{\omega}=E_{\omega} \mathbb{H} .
\end{gathered}
$$

For every $\omega \in \sigma(\mathcal{A})$ let $C_{\omega}^{\infty}$ denote the smooth functions in $\mathbb{H}_{\omega}$ - i.e. let $C_{\omega}^{\infty}=\mathbb{H}_{\omega} \cap C^{\infty}\left(\mathbb{T}^{d}\right)$. We will assume that each $C_{\omega}^{\infty}$ is dense in $\mathbb{H}_{\omega}$ and is contained in the domains of $\mathcal{D}$ and $\mathcal{Q}$. This assumption holds because we are working over the periodic domain $\mathbb{T}^{d}$. In general settings it would mean that every smooth eigenfunction of $\mathcal{A}$ must also satisfy any boundary conditions 
associated with $\mathcal{D}$, which is typically is not true. Our periodic setting we have explicit formulas for each $E_{\omega}$, however we will put off using them as long as possible.

The Spectral Decomposition Theorem implies that

$$
e^{-t \mathcal{A}}=\sum_{\omega \in \sigma(\mathcal{A})} e^{-i \omega t} E_{\omega} \quad \text { for every } t \in \mathbb{R} .
$$

If $W \in \mathbb{H}$ has nonzero components only in a finite number of the $\mathbb{H}_{\omega}$ then there are a finite number of nonzero terms in the decomposition

$$
e^{-t \mathcal{A}} W=\sum_{\omega \in \sigma(\mathcal{A})} e^{-i \omega t} E_{\omega} W
$$

If we assume moreover that each of these components is smooth then by using this decomposition in (3.14) we obtain the spectral formulas

$$
\begin{aligned}
\overline{\mathcal{Q}}(W, W) & =\sum_{\omega_{1}, \omega_{2} \in \sigma(\mathcal{A})} E_{\omega_{1}+\omega_{2}} \mathcal{Q}\left(E_{\omega_{1}} W, E_{\omega_{2}} W\right), \\
\overline{\mathcal{D}} W & =\sum_{\omega \in \sigma(\mathcal{A})} E_{\omega} \mathcal{D} E_{\omega} W
\end{aligned}
$$

where we understand that $E_{\omega_{1}+\omega_{2}}=0$ when $\omega_{1}+\omega_{2} \notin \sigma(\mathcal{A})$.

4.2. Bounds on the Averaged Operators. The spectral formulas (4.1) only apply to those $W \in \mathbb{H}$ that have nonzero components in only a finite number of the $\mathbb{H}_{\omega}$, each of which is smooth. Denote this set by $\oplus_{\omega} C_{\omega}^{\infty}$. While this set is dense in $\mathbb{H}$, it must be extended to larger classes of $W$. This is done by continuity once we obtain appropriate bounds on the forms associated with the operators $\overline{\mathcal{Q}}$ and $\overline{\mathcal{D}}$.

We begin by recalling some bounds on the forms associated with the operators $\mathcal{A}$, $\mathcal{Q}$, and $\mathcal{D}$ in terms of norms that are invariant under $e^{-t \mathcal{A}}$. Specifically, we employ the $\mathbb{H}^{s}$ spaces that are the completion of $\oplus_{\omega} C_{\omega}^{\infty}$ in the norms defined for every $s \in \mathbb{R}$ by

$$
\|W\|_{\mathbb{H}^{s}}=\left(\sum_{\xi \in \mathbb{Z}^{d}}\left(1+|\xi|^{2}\right)^{s}|\widehat{W}(\xi)|_{G_{o}}\right)^{\frac{1}{2}}, \quad \widehat{W}(\xi)=\frac{1}{(2 \pi)^{d}} \int_{\mathbb{T}^{d}} e^{-i \xi \cdot x} W(x) \mathrm{d} x .
$$

Here $\widehat{W}(\xi)$ is the Fourier coefficient associated with the wave vector $\xi \in \mathbb{Z}^{d}$.

Lemma 4.1. There there exist positive constants $C_{\mathcal{A}}$ and $C_{\mathcal{D}}$ such that for every $W_{1}, W_{2} \in$ $C^{\infty}\left(\mathbb{T}^{d}\right)$ and every $s^{\prime} \in \mathbb{R}$ one has the bounds

$$
\begin{aligned}
&\left|\left(W_{1} \mid \mathcal{A} W_{2}\right)_{\mathbb{H}}\right| \leq C_{\mathcal{A}}\left\|W_{1}\right\|_{\mathbb{H}^{-s^{\prime}}}\left\|\nabla_{x} W_{2}\right\|_{\mathbb{H}^{s^{\prime}}} . \\
&\left|\left(W_{1} \mid \mathcal{D} W_{2}\right)_{\mathbb{H}}\right| \leq C_{\mathcal{D}}\left\|\nabla_{x} W_{1}\right\|_{\mathbb{H}^{-s^{\prime}}}\left\|\nabla_{x} W_{2}\right\|_{\mathbb{H}^{s^{\prime}}}
\end{aligned}
$$

For every $s>d / 2$ there exist a positive constant $C_{\mathcal{Q}}^{s}$ such that for every $W_{1}, W_{2}, W_{3} \in C^{\infty}\left(\mathbb{T}^{d}\right)$ and every $s^{\prime} \geq 0$ one has the bounds

$$
\begin{aligned}
& \left|\left(W_{1} \mid \mathcal{Q}\left(W_{2}, W_{3}\right)\right)_{\mathbb{H}}\right| \leq C_{\mathcal{Q}}^{s}\left\|\nabla_{x} W_{1}\right\|_{\mathbb{H}^{s}}\left\|W_{2}\right\|_{\mathbb{H}}\left\|W_{3}\right\|_{\mathbb{H}}, \\
& \left|\left(W_{1} \mid \mathcal{Q}\left(W_{2}, W_{3}\right)\right)_{\mathbb{H}}\right| \leq 2^{s^{\prime}} C_{\mathcal{Q}}^{s}\left\|W_{1}\right\|_{\mathbb{H}^{-s^{\prime}}}\left(\left\|\nabla_{x} W_{2}\right\|_{\mathbb{H}^{s^{\prime}}}\left\|W_{3}\right\|_{\mathbb{H}^{s}}+\left\|W_{2}\right\|_{\mathbb{H}^{s}}\left\|\nabla_{x} W_{3}\right\|_{\mathbb{H}^{s^{\prime}}}\right) .
\end{aligned}
$$

Proof. We refer the reader to [6] for similar proofs.

Because the $\mathbb{H}^{s}$ norms are invariant under $e^{-t \mathcal{A}}$ we can prove the following. 
Lemma 4.2. For every $s>d / 2, s^{\prime} \geq 0$, and $W_{1}, W_{2}, W_{3} \in \oplus_{\omega} C_{\omega}^{\infty}$ one has the bounds

$$
\begin{aligned}
&\left|\left(W_{1} \mid \overline{\mathcal{D}} W_{2}\right)_{\mathbb{H}}\right| \leq C_{\mathcal{D}}\left\|\nabla_{x} W_{1}\right\|_{\mathbb{H}^{-s^{\prime}}}\left\|\nabla_{x} W_{2}\right\|_{\mathbb{H}^{s^{\prime}}}, \\
&\left|\left(W_{1} \mid \overline{\mathcal{Q}}\left(W_{2}, W_{3}\right)\right)_{\mathbb{H}}\right| \leq C_{\mathcal{Q}}^{s}\left\|\nabla_{x} W_{1}\right\|_{\mathbb{H}^{s}}\left\|W_{2}\right\|_{\mathbb{H}}\left\|W_{3}\right\|_{\mathbb{H}}, \\
&\left|\left(W_{1} \mid \overline{\mathcal{Q}}\left(W_{2}, W_{3}\right)\right)_{\mathbb{H}}\right| \leq 2^{s^{\prime}} C_{\mathcal{Q}}^{s}\left\|W_{1}\right\|_{\mathbb{H}^{-s^{\prime}}}\left(\left\|\nabla_{x} W_{2}\right\|_{\mathbb{H}^{s^{\prime}}}\left\|W_{3}\right\|_{\mathbb{H}^{s}}+\left\|W_{2}\right\|_{\mathbb{H}^{s}}\left\|\nabla_{x} W_{3}\right\|_{\mathbb{H}^{s^{\prime}}}\right),
\end{aligned}
$$

where $C_{\mathcal{D}}$ and $C_{\mathcal{Q}}^{s}$ are the constants appearing in (4.2) and (4.3) of Lemma 4.1.

Proof. Because the $\mathbb{H}^{s}$ norms are invariant under $e^{-t \mathcal{A}}$, and because $e^{-t \mathcal{A}}$ and $\nabla_{x}$ commute, for every $s \geq 0$ and every $W \in C^{\infty}\left(\mathbb{T}^{d}\right)$ we have

$$
\left\|e^{-t \mathcal{A}} W\right\|_{\mathbb{H}^{s}}=\|W\|_{\mathbb{H}^{s}}, \quad\left\|\nabla_{x} e^{-t \mathcal{A}} W\right\|_{\mathbb{H}^{s}}=\left\|\nabla_{x} W\right\|_{\mathbb{H}^{s}} .
$$

We first prove the bound on $\overline{\mathcal{D}}$ in (4.4). From the definition of $\overline{\mathcal{D}}$ given in (3.14) we see that

$$
\begin{aligned}
\left(W_{1} \mid \overline{\mathcal{D}} W_{2}\right)_{\mathbb{H}} & =\lim _{T \rightarrow \infty} \frac{1}{2 T} \int_{-T}^{T}\left(W_{1} \mid e^{t \mathcal{A}} \mathcal{D} e^{-t \mathcal{A}} W_{2}\right)_{\mathbb{H}} \mathrm{d} t \\
& =\lim _{T \rightarrow \infty} \frac{1}{2 T} \int_{-T}^{T}\left(e^{-t \mathcal{A}} W_{1} \mid \mathcal{D} e^{-t \mathcal{A}} W_{2}\right)_{\mathbb{H}} \mathrm{d} t .
\end{aligned}
$$

Hence, the bound on $\mathcal{D}$ in (4.2) and the invariances (4.5) imply

$$
\begin{aligned}
\left|\left(W_{1} \mid \overline{\mathcal{D}} W_{2}\right)_{\mathbb{H}}\right| & \leq \lim _{T \rightarrow \infty} \frac{1}{2 T} \int_{-T}^{T}\left|\left(e^{-t \mathcal{A}} W_{1} \mid \mathcal{D} e^{-t \mathcal{A}} W_{2}\right)_{\mathbb{H}}\right| \mathrm{d} t \\
& \leq \lim _{T \rightarrow \infty} \frac{C_{\mathcal{D}}}{2 T} \int_{-T}^{T}\left\|\nabla_{x} e^{-t \mathcal{A}} W_{1}\right\|_{\mathbb{H}}\left\|\nabla_{x} e^{-t \mathcal{A}} W_{2}\right\|_{\mathbb{H}} \mathrm{d} t \\
& =\lim _{T \rightarrow \infty} \frac{C_{\mathcal{D}}}{2 T} \int_{-T}^{T}\left\|\nabla_{x} W_{1}\right\|_{\mathbb{H}}\left\|\nabla_{x} W_{2}\right\|_{\mathbb{H}} \mathrm{d} t=C_{\mathcal{D}}\left\|\nabla_{x} W_{1}\right\|_{\mathbb{H}}\left\|\nabla_{x} W_{2}\right\|_{\mathbb{H}} .
\end{aligned}
$$

This proves the bound on $\overline{\mathcal{D}}$ in (4.4).

We now prove the bounds on $\overline{\mathcal{Q}}$ in (4.4). From the definition of $\overline{\mathcal{Q}}$ given in (3.14) we see that

$$
\begin{aligned}
\left(W_{1} \mid \overline{\mathcal{Q}}\left(W_{2}, W_{3}\right)\right)_{\mathbb{H}} & =\lim _{T \rightarrow \infty} \frac{1}{2 T} \int_{-T}^{T}\left(W_{1} \mid e^{t \mathcal{A}} \mathcal{Q}\left(e^{-t \mathcal{A}} W_{2}, e^{-t \mathcal{A}} W_{3}\right)\right)_{\mathbb{H}} \mathrm{d} t \\
& =\lim _{T \rightarrow \infty} \frac{1}{2 T} \int_{-T}^{T}\left(e^{-t \mathcal{A}} W_{1} \mid \mathcal{Q}\left(e^{-t \mathcal{A}} W_{2}, e^{-t \mathcal{A}} W_{3}\right)\right)_{\mathbb{H}} \mathrm{d} t
\end{aligned}
$$

Hence, the first bound on $\mathcal{Q}$ in (4.3) and the invariances (4.5) imply

$$
\begin{aligned}
\left|\left(W_{1} \mid \overline{\mathcal{Q}}\left(W_{2}, W_{3}\right)\right)_{\mathbb{H}}\right| & \leq \lim _{T \rightarrow \infty} \frac{1}{2 T} \int_{-T}^{T}\left|\left(e^{-t \mathcal{A}} W_{1} \mid \mathcal{Q}\left(e^{-t \mathcal{A}} W_{2}, e^{-t \mathcal{A}} W_{3}\right)\right)_{\mathbb{H}}\right| \mathrm{d} t \\
& \leq \lim _{T \rightarrow \infty} \frac{C_{\mathcal{Q}}^{s}}{2 T} \int_{-T}^{T}\left\|\nabla_{x} e^{-t \mathcal{A}} W_{1}\right\|_{\mathbb{H} s}\left\|e^{-t \mathcal{A}} W_{2}\right\|_{\mathbb{H}}\left\|e^{-t \mathcal{A}} W_{3}\right\|_{\mathbb{H}} \mathrm{d} t \\
& =\lim _{T \rightarrow \infty} \frac{C_{\mathcal{Q}}^{s}}{2 T} \int_{-T}^{T}\left\|\nabla_{x} W_{1}\right\|_{\mathbb{H} s}\left\|W_{2}\right\|_{\mathbb{H}}\left\|W_{3}\right\|_{\mathbb{H}} \mathrm{d} t=C_{\mathcal{Q}}^{s}\left\|\nabla_{x} W_{1}\right\|_{\mathbb{H}^{s}}\left\|W_{2}\right\|_{\mathbb{H}}\left\|W_{3}\right\|_{\mathbb{H}} .
\end{aligned}
$$

This proves the first bound on $\overline{\mathcal{Q}}$ in (4.4). The second bound on $\overline{\mathcal{Q}}$ in (4.4) follows similarly from the second bound on $\mathcal{Q}$ in (4.3) and the invariances (4.5), thereby proving Lemma 4.2. 
4.3. Averaged Quadratic Convection Operator. Until now the entropy has played no role in our analysis of the weakly nonlinear-dissipative approximation. It will now play a central role. Specifically, the fact system (2.1) has a strictly convex entropy implies that its flux $F(U)$ has a special form $[7,16]$. We will use this form to show that the averaged quadratic convection operator satisfies a cyclic identity and some new bounds.

4.3.1. Special Form of the Flux. Henceforth we will assume that the hyperbolic-parabolic system (2.1) has a strictly convex entropy that is thrice continuously differentiable over the convex domain $\mathcal{U}$. Define the set $\mathcal{V}=\left\{H_{U}(U): U \in \mathcal{U}\right\}$. One can show that $\mathcal{V} \subset \mathbb{R}^{\mathrm{N}}$ is a domain and that the mapping $H_{U}: \mathcal{U} \rightarrow \mathcal{V}$ is a diffeomorphism. For every $V \in \mathcal{V}$ we then define $H^{*}(V) \in \mathbb{R}$ and $J^{*}(V) \in \mathbb{R}^{d}$ by

$$
H^{*}(V)=V^{T} U-H(U), \quad J^{*}(V)=V^{T} F(U)-J(U),
$$

where $U \in \mathcal{U}$ is uniquely determined by $H_{U}(U)=V$. Because the entropy flux $J(U)$ is related to $H(U)$ and $F(U)$ by $(2.5)$, one can show that the mappings $H^{*}: \mathcal{V} \rightarrow \mathbb{R}$ and $J^{*}: \mathcal{V} \rightarrow \mathbb{R}^{d}$ are continuously differentiable with

$$
H_{V}^{*}(V)=U, \quad J_{V}^{*}(V)=F(U) .
$$

It follows that $F(U)$ is given by

$$
F(U)=J_{V}^{*}\left(H_{U}(U)\right) \text { for every } U \in \mathcal{U} .
$$

Because we have assumed that $H$ is thrice continuously differentiable while $F$ is twice continuously differentiable over $\mathcal{U}$, it follows from (4.7) that both $H^{*}$ and $J^{*}$ are thrice continuously differentiable over $\mathcal{V}$. This contrasts with $J$, which is twice continuously differentiable over $\mathcal{U}$.

4.3.2. Cyclic Identity. The fact that the flux $F(U)$ of system (2.1) has the form (4.7) is central to our proof of the following identity.

Lemma 4.3. (Cyclic Identity.) If system (2.1) considered over the spatial domain $\mathbb{T}^{d}$ has a strictly convex entropy $H$ satisfying (2.4) that is thrice continuously differentiable over $\mathcal{U}$ then for every $W_{1}, W_{2}, W_{3} \in C^{\infty}\left(\mathbb{T}^{d}\right)$ one has the cyclic identity

$$
\left(W_{1} \mid \overline{\mathcal{Q}}\left(W_{2}, W_{3}\right)\right)_{\mathbb{H}}+\left(W_{2} \mid \overline{\mathcal{Q}}\left(W_{3}, W_{1}\right)\right)_{\mathbb{H}}+\left(W_{3} \mid \overline{\mathcal{Q}}\left(W_{1}, W_{2}\right)\right)_{\mathbb{H}}=0
$$

where $\overline{\mathcal{Q}}$ is defined by (3.14).

Proof. We will first establish (4.9) for the particular $\overline{\mathcal{Q}}$ associated with the conserved densities. In that case $\mathcal{W}=\mathcal{U}$. The extension of (4.9) to general $\overline{\mathcal{Q}}$ then follows from the change of variable formula (3.18).

We will begin by establishing (4.9) when $U_{1}, U_{2}, U_{3} \in \oplus_{\omega} C_{\omega}^{\infty}$. Once this is done, the extension of (4.9) to $U_{1}, U_{2}, U_{3} \in C^{\infty}\left(\mathbb{T}^{d}\right)$ then follows by a density argument that uses the bounds on $\overline{\mathcal{Q}}$ from Lemma 4.2.

Let $U_{1}, U_{2}, U_{3} \in \oplus_{\omega} C_{\omega}^{\infty}$. From the definition of $\overline{\mathcal{Q}}$ given by (3.14) we see that

$$
\begin{aligned}
\left(U_{1} \mid \overline{\mathcal{Q}}\left(U_{2}, U_{3}\right)\right)_{\mathbb{H}} & =\lim _{T \rightarrow \infty} \frac{1}{2 T} \int_{-T}^{T}\left(U_{1} \mid e^{t \mathcal{A}} \mathcal{Q}\left(e^{-t \mathcal{A}} U_{2}, e^{-t \mathcal{A}} U_{3}\right)\right)_{\mathbb{H}} \mathrm{d} t \\
& =\lim _{T \rightarrow \infty} \frac{1}{2 T} \int_{-T}^{T}\left(\widetilde{U}_{1}(t) \mid \mathcal{Q}\left(\widetilde{U}_{2}(t), \widetilde{U}_{3}(t)\right)\right)_{\mathbb{H}} \mathrm{d} t .
\end{aligned}
$$

where $\widetilde{U}_{1}(t)=e^{-t \mathcal{A}} U_{1}, \widetilde{U}_{2}(t)=e^{-t \mathcal{A}} U_{2}$, and $\widetilde{U}_{3}(t)=e^{-t \mathcal{A}} U_{3}$ are quasiperiodic functions of $t$. 
By (3.12) and (3.14) we see that

$$
\left(\widetilde{U}_{1} \mid \mathcal{Q}\left(\widetilde{U}_{2}, \widetilde{U}_{3}\right)\right)_{\mathbb{H}}=\int_{\mathbb{T}^{d}} \widetilde{U}_{1}^{T} G_{o} \nabla_{x} \cdot Q_{o}\left(\widetilde{U}_{2}, \widetilde{U}_{3}\right) \mathrm{d} x=-\int_{\mathbb{T}^{d}} \nabla_{x} \widetilde{U}_{1}^{T} \cdot G_{o} Q_{o}\left(\widetilde{U}_{2}, \widetilde{U}_{3}\right) \mathrm{d} x,
$$

where $G_{o}=H_{U U}\left(U_{o}\right)$ and $Q_{o}\left(\widetilde{U}_{2}, \widetilde{U}_{3}\right)=\frac{1}{2} F_{U U}\left(U_{o}\right)\left(\widetilde{U}_{2} \otimes \widetilde{U}_{3}\right)$. By differentiating (4.8) twice and evaluating at $U_{o}$ we obtain

$$
\begin{aligned}
F_{U}\left(U_{o}\right) & =J_{V V}^{*}\left(V_{o}\right) G_{o} \\
F_{U U}\left(U_{o}\right)\left(\widetilde{U}_{2} \otimes \widetilde{U}_{3}\right) & =J_{V V V}^{*}\left(V_{o}\right)\left(G_{o} \widetilde{U}_{2} \otimes G_{o} \widetilde{U}_{3}\right)+J_{V V}^{*}\left(V_{o}\right) H_{U U U}\left(U_{o}\right)\left(\widetilde{U}_{2} \otimes \widetilde{U}_{3}\right) .
\end{aligned}
$$

where $V_{o}=H_{U}\left(U_{o}\right)$. Hence,

$$
\mathcal{A}=A_{o} \cdot \nabla_{x}=J_{V V}^{*}\left(V_{o}\right) G_{o} \cdot \nabla_{x}
$$

and

$$
\begin{aligned}
\nabla_{x} \widetilde{U}_{1}^{T} \cdot G_{o} Q_{o}\left(\widetilde{U}_{2}, \widetilde{U}_{3}\right)= & \frac{1}{2} \nabla_{x} \widetilde{U}_{1}^{T} \cdot G_{o} J_{V V V}^{*}\left(V_{o}\right)\left(G_{o} \widetilde{U}_{2} \otimes G_{o} \widetilde{U}_{3}\right) \\
& +\frac{1}{2} \nabla_{x} \widetilde{U}_{1}^{T} \cdot G_{o} J_{V V}^{*}\left(V_{o}\right) H_{U U U}\left(U_{o}\right)\left(\widetilde{U}_{2} \otimes \widetilde{U}_{3}\right) \\
= & \frac{1}{2} J_{V V V}^{*}\left(V_{o}\right)\left(G_{o} \cdot \nabla_{x} \widetilde{U}_{1} \otimes G_{o} \widetilde{U}_{2} \otimes G_{o} \widetilde{U}_{3}\right)+\frac{1}{2} H_{U U U}\left(U_{o}\right)\left(\partial_{t} \widetilde{U}_{1} \otimes \widetilde{U}_{2} \otimes \widetilde{U}_{3}\right) .
\end{aligned}
$$

Summing the above relation with its cyclic permutations gives

$$
\begin{aligned}
& \nabla_{x} \widetilde{U}_{1}^{T} \cdot G_{o} Q_{o}\left(\widetilde{U}_{2}, \widetilde{U}_{3}\right)+\nabla_{x} \widetilde{U}_{2}^{T} \cdot G_{o} Q_{o}\left(\widetilde{U}_{3}, \widetilde{U}_{1}\right)+\nabla_{x} \widetilde{U}_{3}^{T} \cdot G_{o} Q_{o}\left(\widetilde{U}_{1}, \widetilde{U}_{2}\right) \\
& =\frac{1}{2} \nabla_{x} \cdot\left[J_{V V V}^{*}\left(V_{o}\right)\left(G_{o} \widetilde{U}_{1} \otimes G_{o} \widetilde{U}_{2} \otimes G_{o} \widetilde{U}_{3}\right)\right]+\frac{1}{2} \partial_{t}\left[H_{U U U}\left(U_{o}\right)\left(\widetilde{U}_{1} \otimes \widetilde{U}_{2} \otimes \widetilde{U}_{3}\right)\right] .
\end{aligned}
$$

Integrating this over $\mathbb{T}^{d}$ we obtain

$$
\begin{aligned}
& \left(\widetilde{U}_{1} \mid \mathcal{Q}\left(\widetilde{U}_{2}, \widetilde{U}_{3}\right)\right)_{\mathbb{H}}+\left(\widetilde{U}_{2} \mid \mathcal{Q}\left(\widetilde{U}_{3}, \widetilde{U}_{1}\right)\right)_{\mathbb{H}}+\left(\widetilde{U}_{3} \mid \mathcal{Q}\left(\widetilde{U}_{1}, \widetilde{U}_{2}\right)\right)_{\mathbb{H}} \\
& =-\frac{1}{2} \frac{\mathrm{d}}{\mathrm{d} t} \int_{\mathbb{T}^{d}} H_{U U U}\left(U_{o}\right)\left(\widetilde{U}_{1} \otimes \widetilde{U}_{2} \otimes \widetilde{U}_{3}\right) \mathrm{d} x
\end{aligned}
$$

The time average of this equation yields

$$
\left(U_{1} \mid \overline{\mathcal{Q}}\left(U_{2}, U_{3}\right)\right)_{\mathbb{H}}+\left(U_{2} \mid \overline{\mathcal{Q}}\left(U_{3}, U_{1}\right)\right)_{\mathbb{H}}+\left(U_{3} \mid \overline{\mathcal{Q}}\left(U_{1}, U_{2}\right)\right)_{\mathbb{H}}=0
$$

because the time average of the time derivative of a bounded function vanishes, thereby proving the lemma.

The cyclic identity (4.9) yields the following bound.

Lemma 4.4. If system (2.1) considered over the spatial domain $\mathbb{T}^{d}$ has a strictly convex entropy $H$ satisfying (2.4) that is thrice continuously differentiable over $\mathcal{U}$ then for every $s>d / 2$ and every $W_{1}, W_{2} \in C^{\infty}\left(\mathbb{T}^{d}\right)$ one has the bound

$$
\left|\left(W_{1} \mid \overline{\mathcal{Q}}\left(W_{1}, W_{2}\right)\right)_{\mathbb{H}}\right| \leq \frac{1}{2} C_{\mathcal{Q}}^{s}\left\|W_{1}\right\|_{\mathbb{H}}^{2}\left\|\nabla_{x} W_{2}\right\|_{\mathbb{H}^{s}},
$$

where $\overline{\mathcal{Q}}\left(W_{1}, W_{2}\right)$ is defined by (3.14) and $C_{\mathcal{Q}}^{s}$ is the constant appearing in (4.3) of Lemma 4.1.

Proof. The cyclic identity (4.9) and the fact that $\overline{\mathcal{Q}}\left(W_{1}, W_{2}\right)=\overline{\mathcal{Q}}\left(W_{2}, W_{1}\right)$ imply

$$
2\left(W_{1} \mid \overline{\mathcal{Q}}\left(W_{1}, W_{2}\right)\right)_{\mathbb{H}}+\left(W_{2} \mid \overline{\mathcal{Q}}\left(W_{1}, W_{1}\right)\right)_{\mathbb{H}}=0 .
$$

It thereby follows from the second bound in (4.4) that

$$
\left|\left(W_{1} \mid \overline{\mathcal{Q}}\left(W_{1}, W_{2}\right)\right)_{\mathbb{H}}\right| \leq \frac{1}{2}\left|\left(W_{2} \mid \overline{\mathcal{Q}}\left(W_{1}, W_{1}\right)\right)_{\mathbb{H}}\right| \leq \frac{1}{2} C_{\mathcal{Q}}^{s}\left\|W_{1}\right\|_{\mathbb{H}}^{2}\left\|\nabla_{x} W_{2}\right\|_{\mathbb{H}^{s}},
$$

where $C_{\mathcal{Q}}^{s}$ is the constant appearing in (4.3) of Lemma 4.1. 
4.4. Averaged Dissipation Operator. Because the averaged dissipation operator $\overline{\mathcal{D}}$ is nonpositive semidefinite, its Friedrichs extension is a self adjoint nonpositive semidefinite operator with domain $\operatorname{Dom}(\overline{\mathcal{D}})$. For every $V \in \mathbb{H}$ and every $\omega \in \sigma(\mathcal{A})$ define $V_{\omega}$ to be the component of $V$ in $\mathbb{H}_{\omega}$ - so that

$$
V_{\omega}=E_{\omega} V \in \mathbb{H}_{\omega}, \quad V=\sum_{\omega \in \sigma(\mathcal{A})} V_{\omega}
$$

We claim that the nonpositve definite Hermitian form associated with $\overline{\mathcal{D}}$ extends to the domain $\operatorname{Herm}(\overline{\mathcal{D}})$ given by

$$
\operatorname{Herm}(\overline{\mathcal{D}})=\left\{V \in \mathbb{H}:-\sum_{\omega \in \sigma(\mathcal{A})}\left(V_{\omega} \mid \mathcal{D} V_{\omega}\right)_{\mathbb{H}}<\infty\right\}
$$

Recall that $V_{\omega} \in \operatorname{Dom}(\mathcal{D})$ for every $\omega \in \sigma(\mathcal{A})$ and that $\mathcal{D}$ is nonpositive over $\operatorname{Dom}(\mathcal{D})$, whereby $\left(V_{\omega} \mid \mathcal{D} V_{\omega}\right)_{\mathbb{H}} \leq 0$ for every $\omega \in \sigma(\mathcal{A})$. Then for every $V \in \operatorname{Herm}(\overline{\mathcal{D}})$ one has by (4.1) that

$$
(V \mid \overline{\mathcal{D}} V)_{\mathbb{H}}=\sum_{\omega \in \sigma(\mathcal{A})}\left(V_{\omega} \mid \mathcal{D} V_{\omega}\right)_{\mathbb{H}} \leq 0
$$

We now characterize when the Hermitian form associated with $\overline{\mathcal{D}}$ is nondegenerate.

\section{Lemma 4.5.}

$$
(V \mid \overline{\mathcal{D}} V)_{\mathbb{H}}<0 \quad \text { for every nonconstant } V \in \operatorname{Herm}(\mathcal{D})
$$

if and only if the Kawashima condition (3.7) holds.

Remark. This kind of characterization was first proved by Kawashima [23, 24].

Proof. First we show that (4.14) implies the Kawashima condition (3.7) holds. Suppose not. Then there exists a nonconstant $V \in \mathbb{H}$ such that $V \in \mathbb{H}_{\omega}$ for some $\omega \in \sigma(\mathcal{A})$ and $V \in \operatorname{Null}(\mathcal{D})$. But then $V=V_{\omega} \in \operatorname{Herm}(\overline{\mathcal{D}})$ with

$$
(V \mid \overline{\mathcal{D}} V)_{\mathbb{H}}=\left(V_{\omega} \mid \mathcal{D} V_{\omega}\right)_{\mathbb{H}}=(V \mid \mathcal{D} V)_{\mathbb{H}}=0,
$$

which contradicts (4.14). Therefore (4.14) implies the Kawashima condition (3.7) holds.

Next we show that the Kawashima condition (3.7) implies (4.14). Suppose that (4.14) is false. Then there exists a nonconstant $V \in \operatorname{Herm}(\overline{\mathcal{D}})$ such that

$$
0=(V \mid \overline{\mathcal{D}} V)_{\mathbb{H}}=\sum_{\omega \in \sigma(\mathcal{A})}\left(V_{\omega} \mid \mathcal{D} V_{\omega}\right)_{\mathbb{H}} .
$$

However this is equivalent to

$$
\left(V_{\omega} \mid \mathcal{D} V_{\omega}\right)_{\mathbb{H}}=0 \quad \text { for every } \omega \in \sigma(\mathcal{A}),
$$

which is equivalent to

$$
V_{\omega} \in \operatorname{Null}(\mathcal{D}) \text { for every } \omega \in \sigma(\mathcal{A}),
$$

But $V \neq 0$ implies that $V_{\omega} \neq 0$ for some $\omega \in \sigma(\mathcal{A})$. But then for this $\omega$ we have $V_{\omega}$ is nonconstant, $V_{\omega} \in \mathbb{H}_{\omega}$, and $V_{\omega} \in \operatorname{Null}(\mathcal{D})$, which contradicts the Kawashima condition (3.7). Therefore the Kawashima condition (3.7) implies (4.14), and the proof of Lemma 4.5 is complete. 


\section{Strictly Dissipative Approximations}

The global existence theory presented in the next section will require that $\overline{\mathcal{D}}$ be strictly elliptic. When this is the case we say that the WND approximation is strictly dissipative. For many physical systems the WND approximation has this property. Indeed, the averaged dissipation operator has played an important role in the study of large time behavior of solutions to hyperbolic-parabolic systems of conservations laws and discrete Boltzmann equations. Kawashima $[25,26]$ showed that in one space dimension the averaged dissipation operator was strictly dissipative whenever the Kawashima condition (3.7) holds. In that case he showed that solutions of the original system are well approximated by solutions to an "effective artificial viscosity" system constructed using the averaged dissipation operator. Motivated by this idea, Hoff and Zumbrun [18, 19] studied multi-dimensional diffusion waves for the barotropic NavierStokes system through an artificial viscosity system constructed with the averaged dissipation operator. Recently, Bianchini-Hanouzet-Natalini [4] used the same idea to study the large-time behavior of smooth solutions for partially dissipative hyperbolic systems with a convex entropy. In each case the averaged dissipation operator was shown to be strictly dissipative through a detailed spectral analysis.

To our knowledge there is no proof that the Kawashima condition (3.7) implies that the averaged dissipation operator is strictly dissipative in multidimensional settings. Here we give a stronger criterion that does the job in our spatially periodic setting. In the Fourier representation we have

$$
\widehat{\mathcal{A V}}(\xi)=i A_{o}(\xi) \widehat{V}(\xi), \quad \widehat{\mathcal{D} V}(\xi)=-B_{o}(\xi) \widehat{V}(\xi)
$$

where $A_{o}(\xi)$ and $B_{o}(\xi)$ are the families of $G_{o}$-symmetric matrices in $\mathbb{R}^{\mathrm{N} \times \mathrm{N}}$ defined for every $\xi \in \mathbb{R}^{d}$ by

One then has

$$
A_{o}(\xi)=\xi \cdot A_{o}, \quad B_{o}(\xi)=\xi^{\otimes 2}: B_{o}
$$

whereby

$$
\widehat{e^{-t \mathcal{A} V}}(\xi)=e^{-i t A_{o}(\xi)} \widehat{V}(\xi)
$$

$$
\widehat{\overline{\mathcal{D}} V}(\xi)=\widehat{\overline{\mathcal{D}}}(\xi) \widehat{V}(\xi), \quad \widehat{\overline{\mathcal{D}}}(\xi)=\lim _{T \rightarrow \infty} \frac{1}{2 T} \int_{-T}^{T} e^{i t A_{o}(\xi)} B_{o}(\xi) e^{-i t A_{o}(\xi)} \mathrm{d} t .
$$

Our Kawashima-type criterion for strict dissipativity is given by the following.

Theorem 5.1. Suppose that for some $\alpha>0$ there exists $\beta>0$ such that

$$
G_{o} B_{o}(\widehat{\xi})+\frac{1}{\alpha^{2}} A_{o}(\widehat{\xi})^{T} G_{o} B_{o}(\widehat{\xi}) A_{o}(\widehat{\xi}) \geq \beta G_{o} \quad \text { for every } \widehat{\xi} \in \mathbb{S}^{d-1} \text {. }
$$

Then there exists $\delta>0$ such that

$$
-(V \mid \overline{\mathcal{D}} V)_{\mathbb{H}} \geq \delta\left\|\nabla_{x} V\right\|_{\mathbb{H}}^{2} \quad \text { for every } V \in C^{2}\left(\mathbb{T}^{d}\right) .
$$

Remark. The Kawashima condition is satisfied whenever (5.1) holds.

Our proof of Theorem 5.1 requires the following lemma.

Lemma 5.1. Let $G \in \mathbb{C}^{\mathrm{N} \times \mathrm{N}}$ be Hermitian positive definite (i.e. $G=G^{*}>0$ ). Let $A \in \mathbb{C}^{\mathrm{N} \times \mathrm{N}}$ be G-Hermitian (i.e. $G A=A^{*} G$ ) and $B \in \mathbb{C}^{\mathrm{N} \times \mathrm{N}}$ be $G$-Hermitian nonnegative definite (i.e. $\left.G B=B^{*} G \geq 0\right)$. Let $C_{A}$ and $C_{B}$ be constants such that $\|A\|_{G} \leq C_{A}$ and $\|B\|_{G} \leq C_{B}$. Suppose that for some $\alpha>0$ there exists $\beta>0$ such that

$$
G B+\frac{1}{\alpha^{2}} A^{*} G B A \geq \beta G .
$$


Then there exists $\delta>0$ depending only on $\alpha, \beta, C_{A}$, and $C_{B}$, such that

$$
\lim _{T \rightarrow \infty} \frac{1}{2 T} \int_{-T}^{T} e^{i t A^{*}} G B e^{-i t A} \mathrm{~d} t \geq \delta G .
$$

Proof. Let $v \in \mathbb{C}^{\mathrm{N}}$ be nonzero and define

$$
f(t)=v^{*} e^{i t A^{*}} G B e^{-i t A} v .
$$

This is a smooth, nonnegative, quasiperiodic, real-valued function that will vanish whenever $e^{-i t A} v$ is a null vector of $B$. In order to prove (5.4) we must obtain a lower bound for its average. The idea of the proof is to show that $f(t)$ cannot be too small for long.

The first two derivatives of $f(t)$ are

$$
\begin{aligned}
& \dot{f}(t)=i v^{*} e^{i t A^{*}}\left(A^{*} G B-G B A\right) e^{-i t A} v, \\
& \ddot{f}(t)=v^{*} e^{i t A^{*}}\left(2 A^{*} G B A-A^{* 2} G B-G B A^{2}\right) e^{-i t A} v .
\end{aligned}
$$

Roughly speaking, we will show that $\ddot{f}(t)$ is dominated by its first term when $f(t)$ is small. For every $\eta>0$ one has

$$
\left|v^{*} e^{i t A^{*}}\left(A^{* 2} G B+G B A^{2}\right) e^{-i t A} v\right| \leq \frac{1}{\eta^{2}} v^{*} e^{i t A^{*}} G B e^{-i t A} v+\eta^{2} v^{*} e^{i t A^{*}} A^{* 2} G B A^{2} e^{-i t A} v,
$$

The first term on the right-hand side above is just $f(t) / \eta^{2}$ while the second can be bounded by $\eta^{2} C_{A}^{4} C_{B} v^{*} G v$. If we set $\eta^{2}=\alpha^{2} \beta /\left(2 C_{A}^{4} C_{B}\right)$ then we obtain the bound

$$
\left|v^{*} e^{i t A^{*}}\left(A^{* 2} G B+G B A^{2}\right) e^{-i t A} v\right| \leq \frac{2 C_{A}^{4} C_{B}}{\alpha^{2} \beta} f(t)+\frac{\alpha^{2} \beta}{2} v^{*} G v .
$$

Let $\Omega^{\epsilon}=\left\{t \in \mathbb{R}: f(t)<\epsilon v^{*} G v\right\}$, where $\epsilon>0$ satisfies

$$
\epsilon \leq \frac{\alpha^{4} \beta}{\alpha^{4} \beta+C_{A}^{4} C_{B}} \frac{\beta}{4} .
$$

For every $t \in \Omega^{\epsilon}$ we obtain from (5.3), (5.5), (5.6), and (5.7) the lower bound

$$
\begin{aligned}
\ddot{f}(t) & \geq v^{*} e^{i t A^{*}}\left(2 \alpha^{2} G B+2 A^{*} G B A\right) e^{-i t A} v-\left(2 \alpha^{2}+\frac{2 C_{A}^{4} C_{B}}{\alpha^{2} \beta}\right) f(t)-\frac{\alpha^{2} \beta}{2} v^{*} G v \\
& \geq\left(2 \alpha^{2} \beta-\left(2 \alpha^{2}+\frac{2 C_{A}^{4} C_{B}}{\alpha^{2} \beta}\right) \epsilon-\frac{\alpha^{2} \beta}{2}\right) v^{*} G v \\
& \geq \alpha^{2} \beta v^{*} G v .
\end{aligned}
$$

For every $t \in \Omega^{\epsilon}$ we obtain from (5.5), (5.6), and (5.7) the upper bound

$$
\begin{aligned}
\ddot{f}(t) & \leq v^{*} e^{i t A^{*}}\left(2 A^{*} G B A\right) e^{-i t A} v+\frac{2 C_{A}^{4} C_{B}}{\alpha^{2} \beta} f(t)+\frac{\alpha^{2} \beta}{2} v^{*} G v \\
& \leq\left(2 C_{A}^{2} C_{B}+\frac{2 C_{A}^{4} C_{B}}{\alpha^{2} \beta} \epsilon+\frac{\alpha^{2} \beta}{2}\right) v^{*} G v \\
& \leq\left(2 C_{A}^{2} C_{B}+\alpha^{2} \beta\right) v^{*} G v .
\end{aligned}
$$

Because $f$ is continuous the set $\Omega^{\epsilon}$ is open and is therefore a countable union of disjoint open intervals:

$$
\Omega^{\epsilon}=\bigcup_{k \in \mathbb{N}}\left(a_{k}^{\epsilon}, b_{k}^{\epsilon}\right)
$$


Let $(a, b)$ be any one of these intervals. Because $\ddot{f}(t)$ satisfies the lower bound (5.8) while $f(t)<\epsilon v^{*} G v$ for every $t \in(a, b)$, it is clear that the interval $(a, b)$ must be bounded.

We will begin by bounding $b-a$ above and below. Because $f$ is continuous over the bounded interval $[a, b]$, and because $a$ and $b$ are not in $\Omega^{\epsilon}$, it follows that $f(a)=f(b)=\epsilon v^{*} G v$, and that $f$ takes its minimum at a point $t_{o} \in(a, b)$, at which $\dot{f}\left(t_{o}\right)=0$. Then

$$
f(t)=f\left(t_{o}\right)+\int_{t_{o}}^{t} \dot{f}\left(t_{1}\right) \mathrm{d} t_{1}=f\left(t_{o}\right)+\int_{t_{o}}^{t} \int_{t_{o}}^{t_{1}} \ddot{f}\left(t_{2}\right) \mathrm{d} t_{2} \mathrm{~d} t_{1} .
$$

We claim that $b-a$ satisfies the bounds

$$
\frac{\epsilon v^{*} G v-f\left(t_{o}\right)}{2 C_{A}^{2} C_{B}+\alpha^{2} \beta} \leq \frac{1}{8}(b-a)^{2} v^{*} G v \leq \frac{\epsilon v^{*} G v}{\alpha^{2} \beta} .
$$

The upper bound of (5.11) is obtained from (5.10) by using the lower bound (5.8) for $\ddot{f}(t)$. For every $t \in(a, b)$ we have

$$
f(t) \geq f\left(t_{o}\right)+\frac{1}{2}\left(t-t_{o}\right)^{2} \alpha^{2} \beta v^{*} G v .
$$

Evaluating this at $t=a$ and $t=b$ yields

$$
\begin{aligned}
& \epsilon v^{*} G v \geq f\left(t_{o}\right)+\frac{1}{2}\left(t_{o}-a\right)^{2} \alpha^{2} \beta v^{*} G v, \\
& \epsilon v^{*} G v \geq f\left(t_{o}\right)+\frac{1}{2}\left(b-t_{o}\right)^{2} \alpha^{2} \beta v^{*} G v .
\end{aligned}
$$

Because $f\left(t_{o}\right) \geq 0$ while $\max \left\{\left(t_{o}-a\right)^{2},\left(b-t_{o}\right)^{2}\right\} \geq \frac{1}{4}(b-a)^{2}$, we obtain

$$
\epsilon v^{*} G v \geq \frac{1}{8}(b-a)^{2} \alpha^{2} \beta v^{*} G v,
$$

which yields the upper bound of (5.11).

The lower bound of (5.11) is obtained from (5.10) by using the upper bound (5.9) for $\ddot{f}(t)$. For every $t \in(a, b)$ we have

$$
f(t) \leq f\left(t_{o}\right)+\frac{1}{2}\left(t-t_{o}\right)^{2}\left(2 C_{A}^{2} C_{B}+\alpha^{2} \beta\right) v^{*} G v,
$$

Evaluating this at $t=a$ and $t=b$ yields

$$
\begin{aligned}
& \epsilon v^{*} G v \leq f\left(t_{o}\right)+\frac{1}{2}\left(t_{o}-a\right)^{2}\left(2 C_{A}^{2} C_{B}+\alpha^{2} \beta\right) v^{*} G v, \\
& \epsilon v^{*} G v \leq f\left(t_{o}\right)+\frac{1}{2}\left(b-t_{o}\right)^{2}\left(2 C_{A}^{2} C_{B}+\alpha^{2} \beta\right) v^{*} G v .
\end{aligned}
$$

Because $\min \left\{\left(t_{o}-a\right)^{2},\left(b-t_{o}\right)^{2}\right\} \leq \frac{1}{4}(b-a)^{2}$, we obtain

$$
\epsilon v^{*} G v \leq f\left(t_{o}\right)+\frac{1}{8}(b-a)^{2}\left(2 C_{A}^{2} C_{B}+\alpha^{2} \beta\right) v^{*} G v,
$$

which yields the lower bound of (5.11).

Next, we bound the average of $f(t)$ over $(a, b)$ from below. By again using the lower bound (5.8) for $\ddot{f}(t)$ in (5.10) we obtain

$$
\begin{aligned}
\frac{1}{b-a} \int_{a}^{b} f(t) \mathrm{d} t & \geq f\left(t_{o}\right)+\frac{\left(b-t_{o}\right)^{2}+\left(b-t_{o}\right)\left(t_{o}-a\right)+\left(t_{o}-a\right)^{2}}{6} \alpha^{2} \beta v^{*} G v \\
& \geq f\left(t_{o}\right)+\frac{1}{8}(b-a)^{2} \alpha^{2} \beta v^{*} G v
\end{aligned}
$$

The lower bound of (5.11) then implies

$$
\frac{1}{b-a} \int_{a}^{b} f(t) \mathrm{d} t \geq f\left(t_{o}\right)+\alpha^{2} \beta \frac{\epsilon v^{*} G v-f\left(t_{o}\right)}{2 C_{A}^{2} C_{B}+\alpha^{2} \beta} \geq \frac{\alpha^{2} \beta \epsilon}{2 C_{A}^{2} C_{B}+\alpha^{2} \beta} v^{*} G v
$$


We are now ready to prove (5.4) with $\delta$ given by

$$
\delta=\frac{\alpha^{2} \beta \epsilon}{2 C_{A}^{2} C_{B}+\alpha^{2} \beta} .
$$

For every $T>0$ let $K_{T}^{\epsilon}=\left\{k \in \mathbb{N}:\left(a_{k}^{\epsilon}, b_{k}^{\epsilon}\right) \subset(-T, T)\right\}$ and

$$
\Omega_{T}^{\epsilon}=\bigcup_{k \in K_{T}^{\epsilon}}\left(a_{k}^{\epsilon}, b_{k}^{\epsilon}\right) \text {. }
$$

Then using the fact that $f(t) \geq \epsilon$ over $[-T, T]-\Omega^{\epsilon}$, the lower bound (5.12), and the fact that $\delta$ given by (5.13) satisfies $\delta<\epsilon$, we find

$$
\begin{aligned}
\frac{1}{2 T} \int_{-T}^{T} f(t) \mathrm{d} t & \geq \frac{1}{2 T}\left[\int_{[-T, T]-\Omega^{\epsilon}} f(t) \mathrm{d} t+\sum_{k \in K_{T}^{\epsilon}} \int_{a_{k}}^{b_{k}} f(t) \mathrm{d} t\right] \\
& \geq \frac{1}{2 T}\left[\operatorname{meas}\left([-T, T]-\Omega^{\epsilon}\right) \epsilon+\sum_{k \in K_{T}^{\epsilon}}\left(b_{k}^{\epsilon}-a_{k}^{\epsilon}\right) \delta\right] v^{*} G v \\
& \geq\left[1-\frac{\operatorname{meas}\left([-T, T] \cap \Omega^{\epsilon}-\Omega_{T}^{\epsilon}\right)}{2 T}\right] \delta v^{*} G v .
\end{aligned}
$$

The set $[-T, T] \cap \Omega^{\epsilon}-\Omega_{T}^{\epsilon}$ is contained in the union of the (at most two) disjoint intervals $\left(a_{k}^{\epsilon}, b_{k}^{\epsilon}\right)$ that contain $-T$ and $T$. So its measure is bounded above by twice the upper bound for $b-a$ given by (5.11) - namely, by

$$
\operatorname{meas}\left([-T, T] \cap \Omega^{\epsilon}-\Omega_{T}^{\epsilon}\right) \leq 4\left(\frac{2 \epsilon}{\alpha^{2} \beta}\right)^{\frac{1}{2}} .
$$

Hence, we obtain

$$
\frac{1}{2 T} \int_{-T}^{T} f(t) \mathrm{d} t \geq\left[1-\frac{2}{T}\left(\frac{2 \epsilon}{\alpha^{2} \beta}\right)^{\frac{1}{2}}\right] \delta v^{*} G v .
$$

Letting $T \rightarrow \infty$ in this inequality yields (5.4). The limit of the left-hand side exists because $f(t)$ is quasiperiodic.

We are now ready to prove Theorem 5.1 with the aid of Lemma 5.1.

Proof of Theorem 5.1. First observe that for every nonzero $\xi \in \mathbb{R}^{d}$ we set $\widehat{\xi}=\xi /|\xi|$ and have

$$
\begin{aligned}
-\widehat{\overline{\mathcal{D}}}(\xi) & =\lim _{T \rightarrow \infty} \frac{1}{2 T} \int_{-T}^{T} e^{i t A_{o}(\xi)} B_{o}(\xi) e^{-i t A_{o}(\xi)} \mathrm{d} t \\
& =|\xi|^{2} \lim _{T \rightarrow \infty} \frac{1}{2 T} \int_{-T}^{T} e^{i t|\xi| A_{o}(\widehat{\xi})} B_{o}(\widehat{\xi}) e^{-i t|\xi| A_{o}(\widehat{\xi})} \mathrm{d} t \\
& =|\xi|^{2} \lim _{T \rightarrow \infty} \frac{1}{2 T} \int_{-T}^{T} e^{i t A_{o}(\widehat{\xi})} B_{o}(\widehat{\xi}) e^{-i t A_{o}(\widehat{\xi})} \mathrm{d} t .
\end{aligned}
$$

Then by the fact $A_{o}(\widehat{\xi})$ is $G_{o}$-symmetric we have

$$
\begin{aligned}
-G_{o} \widehat{\overline{\mathcal{D}}}(\xi) & =|\xi|^{2} \lim _{T \rightarrow \infty} \frac{1}{2 T} \int_{-T}^{T} G_{o} e^{i t A_{o}(\widehat{\xi})} B_{o}(\widehat{\xi}) e^{-i t A_{o}(\widehat{\xi})} \mathrm{d} t \\
& =|\xi|^{2} \lim _{T \rightarrow \infty} \frac{1}{2 T} \int_{-T}^{T} e^{i t A_{o}(\widehat{\xi})^{T}} G_{o} B_{o}(\widehat{\xi}) e^{-i t A_{o}(\widehat{\xi})} \mathrm{d} t .
\end{aligned}
$$


We now apply Lemma 5.1 with $G=G_{o}, A=A_{o}(\widehat{\xi}), B=B_{o}(\widehat{\xi})$,

$$
C_{A}=\max \left\{\left\|A_{o}(\widehat{\xi})\right\|_{G_{o}}: \widehat{\xi} \in \mathbb{S}^{d-1}\right\}, \quad C_{B}=\max \left\{\left\|B_{o}(\widehat{\xi})\right\|_{G_{o}}: \widehat{\xi} \in \mathbb{S}^{d-1}\right\} .
$$

We find that for every $\widehat{\xi} \in \mathbb{S}^{d-1}$ we have the lower bound

$$
\lim _{T \rightarrow \infty} \frac{1}{2 T} \int_{-T}^{T} e^{i t A_{o}(\widehat{\xi})^{T}} G_{o} B_{o}(\widehat{\xi}) e^{-i t A_{o}(\widehat{\xi})} \mathrm{d} t \geq \delta G_{o}
$$

where $\delta$ is given by (5.13). Because $\delta$ only depends on $\alpha, \beta, C_{A}$, and $C_{B}$, it is independent of $\widehat{\xi}$. Combining this lower bound with (5.14) yields

$$
-G_{o} \widehat{\overline{\mathcal{D}}}(\xi) \geq|\xi|^{2} \delta G_{o} \quad \text { for every } \xi \in \mathbb{R}^{d} .
$$

Then for every $V \in C^{2}\left(\mathbb{T}^{d}\right)$ the Plancherel identity implies

$$
-(V \mid \overline{\mathcal{D}} V)_{\mathbb{H}}=-\sum_{\xi \in \mathbb{L}^{d^{*}}} \widehat{V}(\xi)^{*} G_{o} \widehat{\mathcal{D}}(\xi) \widehat{V}(\xi) \geq \delta \sum_{\xi \in \mathbb{L}^{d^{*}}}|\xi|^{2} \widehat{V}(\xi)^{*} G_{o} \widehat{V}(\xi)=\delta\left\|\nabla_{x} V\right\|_{\mathbb{H}}^{2} .
$$

But this is (5.2), thereby proving the theorem.

\section{Global Weak Solutions}

The weakly nonlinear-dissipative approximation is

$$
\partial_{t} W+\mathcal{A} W+\overline{\mathcal{Q}}(W, W)=\overline{\mathcal{D}} W .
$$

Following the Leray theory for the incompressible Navier-Stokes system, we will show that if $\overline{\mathcal{D}}$ is strictly dissipative then (6.1) has global weak solutions for all initial data $W^{\text {in }} \in \mathbb{H}$. This result includes the Leray theory, so it cannot be improved easily.

The key to obtaining global solutions in the Leray theory for the incompressible NavierStokes system is a so-called energy estimate. This designation is a bit misleading because, as we shall see, the estimate is better understood as an entropy estimate.

6.1. Notion of Weak Solution. We call $W \in C([0, \infty) ; \mathrm{w}-\mathbb{H}) \cap L_{\text {loc }}^{2}(\mathrm{~d} t ; \mathbb{V})$ a Leray-type weak solution of weakly nonlinear-dissipative approximation (6.1), if $W$ satisfies the following weak form of (6.1):

$$
\begin{aligned}
0= & \left(V \mid W\left(t_{2}\right)\right)_{\mathbb{H}}-\left(V \mid W\left(t_{1}\right)\right)_{\mathbb{H}}-\int_{t_{1}}^{t_{2}}(\mathcal{A} V \mid W(t))_{\mathbb{H}} \mathrm{d} t \\
& -\int_{t_{1}}^{t_{2}} \int_{\mathbb{T}^{d}} \nabla_{x} V \cdot G_{o} \bar{Q}(W(t), W(t)) \mathrm{d} x \mathrm{~d} t+\int_{t_{1}}^{t_{2}} \int_{\mathbb{T}^{d}} \nabla_{x} V \cdot G_{o} \bar{B} \cdot \nabla_{x} W(t) \mathrm{d} x \mathrm{~d} t,
\end{aligned}
$$

for every function $V \in \mathbb{V}$. These solutions satisfy the entropy inequality

$$
\frac{1}{2}\|W(t)\|_{\mathbb{H}}^{2}-\int_{0}^{t}(W \mid \overline{\mathcal{D}} W)_{\mathbb{H}} \mathrm{d} t \leq \frac{1}{2}\left\|W^{\mathrm{in}}\right\|_{\mathbb{H}}^{2} .
$$

Of course, for every sufficiently nice $W$ one has the identities

$$
(W \mid \mathcal{A} W)_{\mathbb{H}}=0, \quad(W \mid \overline{\mathcal{Q}}(W, W))_{\mathbb{H}}=0
$$


6.2. Existence Theorem. The main theorem of this paper, the global existence of Leray-type weak solution to the weakly nonlinear-dissipative approximation (6.1) is as follows.

Theorem 6.1. Let (6.1) be the weakly nonlinear-dissipative approximation about a constant state $U_{o}$ of the hyperbolic-parabolic system (2.1) with a strictly convex entropy (2.4). Suppose that the diffusive operator $\bar{D}$ in (6.1) is strictly dissipative, i.e. that there exists $\delta>0$ such that

$$
-(V \mid \overline{\mathcal{D}} V)_{\mathbb{H}} \geq \delta\left\|\nabla_{x} V\right\|_{\mathbb{H}}^{2} \quad \text { for every } V \in C^{2}\left(\mathbb{T}^{d}\right) .
$$

Then, for every $\widetilde{W^{\text {in }}} \in \mathbb{V}$, there exists a solution $\widetilde{W} \in C([0, \infty) ; w-\mathbb{H}) \cap L_{\mathrm{loc}}^{2}(\mathrm{~d} t ; \mathbb{V})$ satisfying (6.2) and (6.3).

Remark. If condition (5.1) is satisfied then $\overline{\mathcal{D}}$ will satisfy (6.5) and the above theorem insures the existence of at least one global weak solution.

Remark. In Section 7.2, we apply our theory to the Navier-Stokes system of gas dynamics. The resulting averaged system includes the incompressible Navier-Stokes system as a subsystem. The question of uniqueness for system (6.1) is thereby at least as hard as that of uniqueness for weak solutions of the incompressible Navier-Stokes system.

Proof. The strategy for our proof was introduced by Leray in the context of the incompressible Navier-Stokes system [28], see also [27, 36]. It is now classical compactness argument that has since been used to prove existence of global weak solutions for other equations [10]. It proceeds in four steps. We begin by constructing a sequence of approximate solutions. We then show that this sequences is relatively compact, first in some weak topologies and then in a strong topology. Finally, we show that limit points of this sequence satisfy (6.2) and are thereby weak solutions of (6.1). This strategy strikes a balance between the fact that compactness is easier to establish for weaker topologies and the fact that passing to the limit in nonlinear terms requires convergence in a strong topology.

6.2.1. Step 1: Constructing Approximate Solutions. One can construct a sequence of approximation solutions $W_{n}$ by any method that yields a consistent weak formulation and an energy relation. Here we do this with the Galerkin method.

Let $\left\{\mathbb{H}_{n}\right\}_{n=1}^{\infty}$ be a sequence of subspaces of $\mathbb{H}$ such that each $\mathbb{H}_{n}$ lies within $C^{\infty}\left(\mathbb{T}^{d}\right)$, has dimension $n$, and satisfies $\mathbb{H}_{n} \subset \mathbb{H}_{n+1}$. Assume moreover that this sequence is complete. Let $\mathcal{P}_{n}$ denote the orthogonal projection from $\mathbb{H}$ onto $\mathbb{H}_{n}$. Completeness implies that for every $V \in \mathbb{H}$ one has $\mathcal{P}_{n} V \rightarrow V$ as $n \rightarrow \infty$. The Galerkin approximation of dimension $n$ is the system

$$
\partial_{t} W_{n}+\mathcal{P}_{n} \mathcal{A} W_{n}+\mathcal{P}_{n} \overline{\mathcal{Q}}\left(W_{n}, W_{n}\right)=\mathcal{P}_{n} \overline{\mathcal{D}} W_{n},
$$

where $W_{n}$ takes values in $\mathbb{H}_{n}$. This is a system of $n$ ODEs. Its nonlinearities are quadratic, hence locally Lipschitz. The Picard existence theorem insures that system (6.6) has local solutions. Taking inner product with $W_{n}$, and applying the identities in (6.4), we obtain the energy identity:

$$
\frac{1}{2}\left\|W_{n}(t)\right\|_{\mathbb{H}}^{2}-\int_{0}^{t}\left(W_{n} \mid \overline{\mathcal{D}} W_{n}\right)_{\mathbb{H}} \mathrm{d} t^{\prime}=\frac{1}{2}\left\|W_{n}^{\mathrm{in}}\right\|_{\mathbb{H}}^{2},
$$

for every $t>0$. This energy identity immediately implies a global $L^{2}$ bound on the approximate solutions $W_{n}$, which thereby exists for all time. 
6.2.2. Step 2: Establish Weak Compactness. We claim that the approximate solutions $W_{n}$ are relatively compact in $C([0, \infty) ; \mathrm{w}-\mathbb{H}) \cap \mathrm{w}-L_{\text {loc }}^{2}(\mathrm{~d} t ; \mathrm{w}-\mathbb{V})$.

First, from the energy identity (6.7), we that $W_{n}(t)$ is uniformly bounded in $\mathbb{H}$ thus relatively compact in $\mathrm{w}-\mathbb{H}$ for every $t>0$. Next, from the dissipation property of $\overline{\mathcal{D}}$, see (6.5), we have

$$
\delta \int_{0}^{t}\left\|\nabla_{x} W_{n}\right\|_{\mathbb{H}}^{2} \mathrm{~d} t^{\prime} \leq-\int_{0}^{t}\left(W_{n} \mid \overline{\mathcal{D}} W_{n}\right)_{H} \mathrm{~d} t^{\prime} \leq C .
$$

Thus $W_{n}$ is relatively compact in w- $L_{l o c}^{2}([0, \infty) ; \mathrm{w}-\mathbb{V})$. We need only to verify that $W_{n}$ is equicontinuous in $C([0, \infty) ; \mathrm{w}-\mathbb{H})$ thus by Arzela-Ascoli theorem the weak compactness is established. The equicontinuity can be derived from the weak form of the Galerkin system (6.6).

$$
\begin{aligned}
\left(\widetilde{V} \mid W_{n}\left(t_{2}\right)-W_{n}\left(t_{1}\right)\right)_{\mathbb{H}}= & \int_{t_{1}}^{t_{2}}\left(\widetilde{V} \mid \mathcal{A} W_{n}(t)\right)_{\mathbb{H}} \mathrm{d} t \\
& -\int_{t_{1}}^{t_{2}}\left(\widetilde{V} \mid \overline{\mathcal{Q}}\left(W_{n}(t), W_{n}(t)\right)\right)_{\mathbb{H}} \mathrm{d} t+\int_{t_{1}}^{t_{2}}\left(\widetilde{V} \mid \overline{\mathcal{D}} W_{n}(t)\right)_{\mathbb{H}} \mathrm{d} t,
\end{aligned}
$$

for every function $\widetilde{V} \in \mathbb{V}$. We first prove the equicontinuity for test function $\widetilde{V} \in \mathbb{V} \cap C^{1}\left(\mathbb{T}^{d}\right)$, which is followed from the first and third bounds in the Lemma 4.2. Then we extend the class of test functions to $\mathbb{V}$ by standard density argument, thereby finishing the proof of Step 2 .

6.2.3. Step 3: Establish Strong Compactness. We claim that $W_{n}$ is relatively compact in strong topology of $L_{l o c}^{2}([0, \infty) ; \mathbb{H})$. It is a direct consequence of the weak compactness result in Step 2 and the fact that the injection

$$
C([0, \infty) ; \mathrm{w}-\mathbb{H}) \cap \mathrm{w}-L_{\mathrm{loc}}^{2}(\mathrm{~d} t ; \mathrm{w}-\mathbb{V}) \longrightarrow L_{l o c}^{2}([0, \infty) ; \mathbb{H})
$$

is continuous.

6.2.4. Step 4: Pass to the Limit. Step 2 ensures that there is a subsequence of $W_{n}$, which we also refer to as $W_{n}$, converges in $C([0, \infty) ; \mathrm{w}-\mathbb{H}) \cap \mathrm{w}-L_{\text {loc }}^{2}(\mathrm{~d} t ; \mathrm{w}-\mathbb{V})$ to a limit $W \in C([0, \infty) ; \mathrm{w}-\mathbb{H}) \cap$ $L_{\text {loc }}^{2}(\mathrm{~d} t ; \mathbb{V})$. Step 3 ensures the convergence of $W_{n}$ to $W$ in $L_{l o c}^{2}([0, \infty) ; \mathbb{H})$. All that remains is to show that the limit $W$ satisfies the weak form (6.2) as well as the energy inequality (6.3). Toward this end we check convergence of each term in the respective regularized versions, (6.9) and (6.7), respectively. Again, we first consider the test function $V$ in the class $\mathbb{V} \cap C^{1}\left(\mathbb{T}^{d}\right)$ then use density argument later. First

$$
\left(V \mid W_{n}\left(t_{2}\right)-W_{n}\left(t_{1}\right)\right)_{\mathbb{H}} \rightarrow\left(V \mid W\left(t_{2}\right)-W\left(t_{1}\right)\right)_{\mathbb{H}} \quad \text { as } \quad n \rightarrow \infty,
$$

because of the relative compactness of $W_{n}$ in $C([0, \infty)$; w- $\mathbb{H})$. The convergence of the first term on the righthand side of $(6.9)$ is trivial. Note that

$$
\int_{t_{1}}^{t_{2}}\left(V \mid \overline{\mathcal{Q}}\left(W_{n}(t), W_{n}(t)\right)\right)_{\mathbb{H}} \mathrm{d} t=-\int_{t_{1}}^{t_{2}} \int_{\mathbb{T}^{d}} \nabla_{x} V \cdot G_{o} \bar{Q}\left(W_{n}(t), W_{n}(t)\right) \mathrm{d} x \mathrm{~d} t,
$$

and $\bar{Q}\left(W_{n}(t), W_{n}(t)\right)$ is quadratic in $W_{n}$. Thus the strong compactness of $W_{n}$ in $L_{\text {loc }}^{2}([0, \infty) ; \mathbb{H})$ in Step 3 ensures the convergence of above term. We also note that

$$
\int_{t_{1}}^{t_{2}}\left(V \mid \overline{\mathcal{D}} W_{n}(t)\right)_{\mathbb{H}} \mathrm{d} t=\int_{t_{1}}^{t_{2}} \int_{\mathbb{T}^{d}} \nabla_{x} V \cdot G_{o} \bar{B} \cdot \nabla_{x} W_{n}(t) \mathrm{d} x \mathrm{~d} t,
$$

The convergence of above term is straightforward. Thus, we show that the limit $W$ satisfy the weak form (6.2), thus is a weak solution to WND approximation (6.1). 
Now, to recover the energy inequality (6.3) from (6.7), first we note that for the initial data term

$$
\left\|W_{n}^{\text {in }}\right\|_{\mathbb{H}} \rightarrow\left\|W^{\text {in }}\right\|_{\mathbb{H}}
$$

The convergence of $W_{n}$ in $C([0, \infty) ; \mathrm{w}-\mathbb{H})$ and $L_{l o c}^{2}([0, \infty) ; \mathbb{H})$, together with the fact that the norm of the weak limit is an eventual lower bound to the norms of the sequence, yields

$$
\|W\|_{\mathbb{H}}^{2} \leq \liminf _{n \rightarrow \infty}\left\|W_{n}(t)\right\|_{\mathbb{H}}^{2}
$$

Similarly, the convergence of $W_{n}$ in $\mathrm{w}-L_{l o c}^{2}([0, \infty) ; \mathrm{w}-\mathbb{V})$ implies

$$
-\int_{0}^{t}(W \mid \overline{\mathcal{D}} W)_{\mathbb{H}} \mathrm{d} t \leq-\liminf _{n \rightarrow \infty} \int_{0}^{t}\left(W_{n} \mid \overline{\mathcal{D}} W_{n}\right)_{\mathbb{H}} \mathrm{d} t
$$

Thus, we finish the proof of global Leray type weak solutions.

6.3. Uniqueness Theorem. Uniqueness can never be asserted by such a compactness argument, but generally requires the knowledge of additional regularity of the solution. For example, here we will prove the following weak-strong theorem.

Theorem 6.2. Let $U_{1}, U_{2} \in C([0, \infty) ; w-\mathbb{H}) \cap L_{\text {loc }}^{2}(\mathrm{~d} t ; \mathbb{V})$ be two weak solutions of the WND system with initial data $U_{1}^{\text {in }}, U_{2}^{\text {in }} \in \mathbb{H}$. Let $s>\max \{d / 2,1\}$. If $U_{1} \in L^{2}\left([0, T] ; \mathbb{H}^{s}\right) \cap L^{1}\left([0, T] ; \mathbb{V}^{s}\right)$ for some $T>0$ then $U_{1} \in C\left([0, T] ; w-\mathbb{H}^{s-1}\right)$ and for every $t \in[0, T]$ one has the energy equality

$$
\frac{1}{2}\left\|U_{1}(t)\right\|_{\mathbb{H}}^{2}-\int_{0}^{t}\left(U_{1} \mid \overline{\mathcal{D}} U_{1}\right)_{\mathbb{H}} \mathrm{d} t^{\prime}=\frac{1}{2}\left\|U_{1}^{\text {in }}\right\|_{\mathbb{H}}^{2},
$$

and the stability bound

$$
\left\|U_{2}(t)-U_{1}(t)\right\|_{\mathbb{H}} \leq \exp \left(C_{\mathcal{Q}}^{s} \int_{0}^{t}\left\|\nabla_{x} U_{1}(t)\right\|_{\mathbb{H}^{s}} \mathrm{~d} t^{\prime}\right)\left\|U_{2}^{\text {in }}-U_{1}^{\text {in }}\right\|_{\mathbb{H}} .
$$

In particular, if $U_{2}^{\text {in }}=U_{1}^{\text {in }}$ then $U_{2}(t)=U_{1}(t)$ for every $t \in[0, T]$.

Remark. Equation (6.11) is simply the assertion that the energy inequality satisfied by the strong solution $U_{1}$ is in fact an equality. The bound (6.12) is a basic weak-strong stability bound, from which the uniqueness assertion follows immediately.

The key to the proof of Theorem 6.2 will be provided by the following lemma.

Lemma 6.1. Let $U_{1}, U_{2} \in C([0, \infty) ; w-\mathbb{H}) \cap L_{\mathrm{loc}}^{2}(\mathrm{~d} t ; \mathbb{V})$ be two weak solutions of the WND system with initial data $U_{1}^{\text {in }}, U_{2}^{\text {in }} \in \mathbb{H}$. Let $s>\max \{d / 2,1\}$. If $U_{1} \in L^{2}\left([0, T] ; \mathbb{H}^{s}\right) \cap L^{1}\left([0, T] ; \mathbb{V}^{s}\right)$ for some $T>0$ then $U_{1} \in C\left([0, T] ; w-\mathbb{H}^{s-1}\right)$ and for every $t \in[0, T]$ one has

$$
\begin{aligned}
&\left(U_{1}(t) \mid U_{2}(t)\right)_{\mathbb{H}}+\int_{0}^{t}\left(\overline{\mathcal{Q}}\left(U_{1}, U_{1}\right) \mid U_{2}\right)_{\mathbb{H}}+\left(U_{1} \mid \overline{\mathcal{Q}}\left(U_{2}, U_{2}\right)\right)_{\mathbb{H}} \mathrm{d} t^{\prime} \\
&-\int_{0}^{t}\left(\overline{\mathcal{D}} U_{1} \mid U_{2}\right)_{\mathbb{H}}+\left(U_{1} \mid \overline{\mathcal{D}} U_{2}\right)_{\mathbb{H}} \mathrm{d} t^{\prime}=\left(U_{1}^{\mathrm{in}} \mid U_{2}^{\mathrm{in}}\right)_{\mathbb{H}} .
\end{aligned}
$$

This lemma will be proved later. Now we will use it to prove Theorem 6.2.

Proof of Theorem 6.2. The energy equality (6.11) follows by setting $U_{2}=U_{1}$ in equation (6.13) of Lemma 6.1 , using the cyclic identity (4.9) to see that $\left(U_{1} \mid \overline{\mathcal{Q}}\left(U_{1}, U_{1}\right)\right)_{\mathbb{H}}=0$, and multiplying the result by $\frac{1}{2}$. 
We now derive the stability bound (6.12). Add the energy inequalities for $U_{1}$ and $U_{2}$ and subtract equation (6.13) from the result to obtain

$$
\begin{aligned}
\frac{1}{2}\left\|U_{2}(t)-U_{1}(t)\right\|_{\mathbb{H}}^{2}-\int_{0}^{t}\left(\overline{\mathcal{Q}}\left(U_{1}, U_{1}\right) \mid U_{2}\right)_{\mathbb{H}}+\left(U_{1} \mid \overline{\mathcal{Q}}\left(U_{2}, U_{2}\right)\right)_{\mathbb{H}} \mathrm{d} t^{\prime} & \\
& -\int_{0}^{t}\left(\left(U_{2}-U_{1}\right) \mid \overline{\mathcal{D}}\left(U_{2}-U_{1}\right)\right)_{\mathbb{H}} \mathrm{d} t^{\prime} \leq \frac{1}{2}\left\|U_{2}^{\text {in }}-U_{1}^{\text {in }}\right\|_{\mathbb{H}}^{2} .
\end{aligned}
$$

Upon letting $W=U_{2}-U_{1}$ (so that $U_{2}=U_{1}+W$ ), we see that

$$
\begin{aligned}
\left(\overline{\mathcal{Q}}\left(U_{1}, U_{1}\right) \mid U_{2}\right)_{\mathbb{H}}+\left(U_{1} \mid \overline{\mathcal{Q}}\left(U_{2}, U_{2}\right)\right)_{\mathbb{H}}= & \left(U_{1} \mid \overline{\mathcal{Q}}\left(U_{1}, U_{1}\right)\right)_{\mathbb{H}}+\left(W \mid \overline{\mathcal{Q}}\left(U_{1}, U_{1}\right)\right)_{\mathbb{H}} \\
& +\left(U_{1} \mid \overline{\mathcal{Q}}\left(U_{1}, U_{1}\right)\right)_{\mathbb{H}}+\left(U_{1} \mid \overline{\mathcal{Q}}\left(W, U_{1}\right)\right)_{\mathbb{H}} \\
& +\left(U_{1} \mid \overline{\mathcal{Q}}\left(U_{1}, W\right)\right)_{\mathbb{H}}+\left(U_{1} \mid \overline{\mathcal{Q}}(W, W)\right)_{\mathbb{H}} .
\end{aligned}
$$

The cyclic identity (4.9) implies that $\left(U_{1} \mid \overline{\mathcal{Q}}\left(U_{1}, U_{1}\right)\right)_{\mathbb{H}}=0$ and

$$
\left(W \mid \overline{\mathcal{Q}}\left(U_{1}, U_{1}\right)\right)_{\mathbb{H}}+\left(U_{1} \mid \overline{\mathcal{Q}}\left(W, U_{1}\right)\right)_{\mathbb{H}}+\left(U_{1} \mid \overline{\mathcal{Q}}\left(U_{1}, W\right)\right)_{\mathbb{H}}=0
$$

We thereby see that relation (6.15) reduces to

$$
\left(\overline{\mathcal{Q}}\left(U_{1}, U_{1}\right) \mid U_{2}\right)_{\mathbb{H}}+\left(U_{1} \mid \overline{\mathcal{Q}}\left(U_{2}, U_{2}\right)\right)_{\mathbb{H}}=\left(U_{1} \mid \overline{\mathcal{Q}}(W, W)\right)_{\mathbb{H}} .
$$

When this relation is placed into (6.14), we obtain

$$
\frac{1}{2}\|W(t)\|_{\mathbb{H}}^{2}-\int_{0}^{t}\left(U_{1} \mid \overline{\mathcal{Q}}(W, W)\right)_{\mathbb{H}} \mathrm{d} t^{\prime}-\int_{0}^{t}(W \mid \overline{\mathcal{D}} W)_{\mathbb{H}} \mathrm{d} t^{\prime} \leq \frac{1}{2}\left\|W^{\mathrm{in}}\right\|_{\mathbb{H}}^{2},
$$

where $W^{\text {in }}=U_{2}^{\text {in }}-U_{1}^{\text {in }}$. The third bound in (4.4) gives

$$
\left|\left(U_{1} \mid \overline{\mathcal{Q}}(W, W)\right)_{\mathbb{H}}\right| \leq C_{\mathcal{Q}}^{s}\left\|\nabla_{x} U_{1}\right\|_{\mathbb{H}^{s}}\|W\|_{\mathbb{H}}^{2}
$$

We combine this bound with the fact $-(W \mid \overline{\mathcal{D}} W)_{\mathbb{H}} \geq 0$ to see that (6.16) yields the inequality

$$
\frac{1}{2}\|W(t)\|_{\mathbb{H}}^{2} \leq \frac{1}{2}\left\|W^{\mathrm{in}}\right\|_{\mathbb{H}}^{2}+\int_{0}^{t} C_{\mathcal{Q}}^{s}\left\|\nabla_{x} U_{1}\right\|_{\mathbb{H}^{s}}\|W\|_{\mathbb{H}}^{2} \mathrm{~d} t^{\prime}
$$

The stability bound (6.12) then follows by the Gronwall Lemma.

All that remains is to prove Lemma 6.1 .

Proof of Lemma 6.1. We begin by showing that $U_{1} \in L^{2}\left([0, T] ; \mathbb{H}^{s}\right) \cap L^{1}\left([0, T] ; \mathbb{V}^{s}\right)$ implies $U_{1} \in C\left([0, T] ; \mathrm{w}-\mathbb{H}^{s-1}\right)$. From equation (6.1) we see that for every $W \in \mathbb{H}^{-(s-1)}$ we have

$$
\left(W \mid U_{1}\left(t_{2}\right)-U_{1}\left(t_{1}\right)\right)_{\mathbb{H}}=-\int_{t_{1}}^{t_{2}}\left(W \mid \mathcal{A} U_{1}(t)+\overline{\mathcal{Q}}\left(U_{1}(t), U_{1}(t)\right)-\overline{\mathcal{D}} U_{1}(t)\right)_{\mathbb{H}} \mathrm{d} t
$$

We have the following estimates:

$$
\left|\int_{t_{1}}^{t_{2}}\left(W \mid \mathcal{A} U_{1}(t)\right)_{\mathbb{H}} \mathrm{d} t\right| \leq C_{\mathcal{A}}\|W\|_{\mathbb{H}^{-(s-1)}} \int_{t_{1}}^{t_{2}}\left\|U_{1}(t)\right\|_{\mathbb{H}^{s}} \mathrm{~d} t
$$

By the first bound in (4.3) of Lemma 4.2 with $s^{\prime}=s$ we have

$$
\begin{aligned}
\left|\int_{t_{1}}^{t_{2}}\left(W \mid \overline{\mathcal{D}} U_{1}(t)\right)_{\mathbb{H}} \mathrm{d} t\right| & \leq C_{\mathcal{D}}\left\|\nabla_{x} W\right\|_{\mathbb{H}^{-s}} \int_{t_{1}}^{t_{2}}\left\|\nabla_{x} U_{1}(t)\right\|_{\mathbb{H}^{s}} \mathrm{~d} t \\
& \leq C_{\mathcal{D}}\|W\|_{\mathbb{H}^{-(s-1)}} \int_{t_{1}}^{t_{2}}\left\|U_{1}(t)\right\|_{\mathbb{V} s} \mathrm{~d} t .
\end{aligned}
$$


By the third bound in (4.3) of Lemma 4.2 with $s^{\prime}=s-1$ we have

$$
\left|\int_{t_{1}}^{t_{2}}\left(W \mid \overline{\mathcal{Q}}\left(U_{1}(t), U_{1}(t)\right)\right)_{\mathbb{H}} \mathrm{d} t\right| \leq 2^{s} C_{\mathcal{Q}}^{s-1}\|W\|_{\mathbb{H}^{-(s-1)}} \int_{t_{1}}^{t_{2}}\left\|U_{1}(t)\right\|_{\mathbb{H}^{s}}^{2} \mathrm{~d} t
$$

Because $U_{1} \in L^{2}\left([0, T] ; \mathbb{H}^{s}\right) \cap L^{1}\left([0, T] ; \mathbb{V}^{s}\right)$ it follows that $\left(W \mid U_{1}(t)\right)_{\mathbb{H}}$ is a continuous function of $t$ over $[0, T]$ for every $W \in \mathbb{H}^{s-1}$. Hence, $U_{1} \in C\left([0, T] ; \mathrm{w}-\mathbb{H}^{s-1}\right)$.

We now prove that (6.13) holds for every $t \in[0, T]$. Let $\alpha \in \mathcal{D}\left(\mathbb{R}^{d}\right)$ and $\beta \in \mathcal{D}(\mathbb{R})$ be mollifiers such that $\alpha \geq 0, \beta \geq 0, \operatorname{supp}(\beta) \subset(-\infty, 0]$ and

$$
\int_{\mathbb{R}^{d}} \alpha(x) \mathrm{d} x=1, \quad \int_{\mathbb{R}} \beta(t) \mathrm{d} t=1 .
$$

For every $\epsilon>0$ define $\Theta_{\epsilon} \in \mathcal{D}\left(\mathbb{T}^{d} \times \mathbb{R}\right)$ by

$$
\Theta_{\epsilon}(x, t)=\frac{1}{\epsilon^{d+1}} \sum_{l \in \mathbb{Z}^{d}} \alpha\left(\frac{x+2 \pi l}{\epsilon}\right) \beta\left(\frac{t}{\epsilon}\right) .
$$

For each $i=1,2$ and $\epsilon>0$ define $U_{i \epsilon}=\Theta_{\epsilon} * U_{i}$, so that for every $x \in \mathbb{T}^{d}$ and $t \geq 0$ we have

$$
U_{i \epsilon}(x, t)=\left(\Theta_{\epsilon} * U_{i}\right)(x, t)=\int_{0}^{\infty} \int_{\mathbb{T}^{d}} \Theta_{\epsilon}\left(x-x^{\prime}, t-t^{\prime}\right) U_{i}\left(x^{\prime}, t^{\prime}\right) \mathrm{d} x^{\prime} \mathrm{d} t^{\prime} .
$$

Each $U_{i \epsilon}$ is a smooth function over $\mathbb{R}^{d} \times \mathbb{R}_{+}$that satisfies

$$
\partial_{t} U_{i \epsilon}+\mathcal{A} U_{i \epsilon}+\Theta_{\epsilon} * \overline{\mathcal{Q}}\left(U_{i}, U_{i}\right)=\overline{\mathcal{D}} U_{i \epsilon} .
$$

Because $\mathcal{A}$ is skew-adjoint we thereby see that

$$
\begin{aligned}
\frac{\mathrm{d}}{\mathrm{d} t}\left(U_{1 \epsilon} \mid U_{2 \epsilon}\right)_{\mathbb{H}}= & \left(\partial_{t} U_{1 \epsilon} \mid U_{2 \epsilon}\right)_{\mathbb{H}}+\left(U_{1 \epsilon} \mid \partial_{t} U_{2 \epsilon}\right)_{\mathbb{H}} \\
= & -\left(\Theta_{\epsilon} * \overline{\mathcal{Q}}\left(U_{1}, U_{1}\right) \mid U_{2 \epsilon}\right)_{\mathbb{H}}-\left(U_{1 \epsilon} \mid \Theta_{\epsilon} * \overline{\mathcal{Q}}\left(U_{2}, U_{2}\right)\right)_{\mathbb{H}} \\
& +\left(\overline{\mathcal{D}} U_{1 \epsilon} \mid U_{2 \epsilon}\right)_{\mathbb{H}}+\left(U_{1 \epsilon} \mid \overline{\mathcal{D}} U_{2 \epsilon}\right)_{\mathbb{H}} .
\end{aligned}
$$

Upon integrating this equation over $[0, t]$ we obtain

$$
\begin{aligned}
\left(U_{1 \epsilon}(t) \mid U_{2 \epsilon}(t)\right)_{\mathbb{H}} & +\int_{0}^{t}\left(\Theta_{\epsilon} * \overline{\mathcal{Q}}\left(U_{1}, U_{1}\right) \mid U_{2 \epsilon}\right)_{\mathbb{H}}+\left(U_{1 \epsilon} \mid \Theta_{\epsilon} * \overline{\mathcal{Q}}\left(U_{2}, U_{2}\right)\right)_{\mathbb{H}} \mathrm{d} t^{\prime} \\
& -\int_{0}^{t}\left(\overline{\mathcal{D}} U_{1 \epsilon} \mid U_{2 \epsilon}\right)_{\mathbb{H}}+\left(U_{1 \epsilon} \mid \overline{\mathcal{D}} U_{2 \epsilon}\right)_{\mathbb{H}} \mathrm{d} t^{\prime} \\
& =\left(U_{1 \epsilon}(0) \mid U_{2 \epsilon}(0)\right)_{\mathbb{H}} .
\end{aligned}
$$

We claim that

$$
\begin{aligned}
\lim _{\epsilon \rightarrow 0}\left(U_{1 \epsilon}(t) \mid U_{2 \epsilon}(t)\right)_{\mathbb{H}} & =\left(U_{1}(t) \mid U_{2}(t)\right)_{\mathbb{H}}, \\
\lim _{\epsilon \rightarrow 0}\left(U_{1 \epsilon}(0) \mid U_{2 \epsilon}(0)\right)_{\mathbb{H}} & =\left(U_{1}^{\mathrm{in}} \mid U_{2}^{\mathrm{in}}\right)_{\mathbb{H}}, \\
\lim _{\epsilon \rightarrow 0} \int_{0}^{t}\left(\Theta_{\epsilon} * \overline{\mathcal{Q}}\left(U_{1}, U_{1}\right) \mid U_{2 \epsilon}\right)_{\mathbb{H}} \mathrm{d} t^{\prime} & =\int_{0}^{t}\left(\overline{\mathcal{Q}}\left(U_{1}, U_{1}\right) \mid U_{2}\right)_{\mathbb{H}} \mathrm{d} t^{\prime}, \\
\lim _{\epsilon \rightarrow 0} \int_{0}^{t}\left(U_{1 \epsilon} \mid \Theta_{\epsilon} * \overline{\mathcal{Q}}\left(U_{2}, U_{2}\right)\right)_{\mathbb{H}} \mathrm{d} t^{\prime} & =\int_{0}^{t}\left(U_{1} \mid \overline{\mathcal{Q}}\left(U_{2}, U_{2}\right)\right)_{\mathbb{H}} \mathrm{d} t^{\prime}, \\
\lim _{\epsilon \rightarrow 0} \int_{0}^{t}\left(\overline{\mathcal{D}} U_{1 \epsilon} \mid U_{2 \epsilon}\right)_{\mathbb{H}}+\left(U_{1 \epsilon} \mid \overline{\mathcal{D}} U_{2 \epsilon}\right)_{\mathbb{H}} \mathrm{d} t^{\prime} & =\int_{0}^{t}\left(\overline{\mathcal{D}} U_{1} \mid U_{2}\right)_{\mathbb{H}}+\left(U_{1} \mid \overline{\mathcal{D}} U_{2}\right)_{\mathbb{H}} \mathrm{d} t^{\prime} .
\end{aligned}
$$


Once these limits are established we can then pass to the limit in (6.17) to obtain (6.13) and thereby complete the proof of Lemma 6.1. The limits (6.18) are established by using the bounds from Lemma 4.2 and the convergence and boundedness properties of convolution.

Now the first two limits are direct consequence of the convergence property of convolution in $C\left([0, T] ; \mathrm{w}-\mathbb{H}^{s-1}\right)$. Because $U_{1}$ and $U_{2}$ are continuous in time in $\mathrm{w}-\mathbb{H}^{s-1}$ and $\mathbb{H}$ respectively, we have, for any $t>0$,

$$
\begin{aligned}
& U_{1 \epsilon}(t) \rightarrow U_{1}(t) \quad \text { in } \quad \mathrm{w}-\mathbb{H}^{(s-1)} \quad \text { thus } \quad U_{1 \epsilon}(t) \rightarrow U_{1}(t) \quad \text { in } \quad \mathbb{H}, \\
& U_{2 \epsilon}(t) \rightarrow U_{2}(t) \quad \text { in } \quad \mathrm{w}-\mathbb{H},
\end{aligned}
$$

as $\epsilon \rightarrow 0$, which imply that for every $t>0$,

$$
\left(U_{1 \epsilon}(t) \mid U_{2 \epsilon}(t)\right)_{\mathbb{H}} \rightarrow\left(U_{1}(t) \mid U_{2}(t)\right)_{\mathbb{H}}
$$

as $\epsilon \rightarrow 0$. Thus we prove the first two limits.

To prove the third limit, first, we have

$$
\begin{aligned}
& \int_{0}^{t}\left(\Theta_{\epsilon} * \overline{\mathcal{Q}}\left(U_{1}, U_{1}\right) \mid U_{2 \epsilon}\right)_{\mathbb{H}}-\left(\overline{\mathcal{Q}}\left(U_{1}, U_{1}\right) \mid U_{2}\right)_{\mathbb{H}} \mathrm{d} t^{\prime} \\
& =\int_{0}^{t}\left(\Theta_{\epsilon} * \overline{\mathcal{Q}}\left(U_{1}, U_{1}\right)-\overline{\mathcal{Q}}\left(U_{1}, U_{1}\right) \mid U_{2 \epsilon}\right)_{\mathbb{H}} \mathrm{d} t^{\prime}+\int_{0}^{t}\left(\overline{\mathcal{Q}}\left(U_{1}, U_{1}\right) \mid U_{2 \epsilon}-U_{2}\right)_{\mathbb{H}} \mathrm{d} t^{\prime}
\end{aligned}
$$

Note that $U_{1}$ is a strong solution, i.e. $U_{1} \in L^{\infty}\left([0, T] ; \mathbb{H}^{s}\right) \cap L^{2}\left([0, T] ; \mathbb{V}^{s}\right)$ for $s>d / 2$, and the structure of $\overline{\mathcal{Q}}\left(U_{1}, U_{1}\right)$ is the derivative of $U_{1}$ multiplying $U_{1}$, thus

$$
\overline{\mathcal{Q}}\left(U_{1}, U_{1}\right) \in L^{2}([0, T] ; \mathbb{H}) .
$$

Then the convergence in $L^{2}([0, T] ; \mathbb{H})$ and the boundedness of mollifier imply that each integral on the right-hand side of (6.19) goes to 0 as $\epsilon \rightarrow 0$.

We leave the proof of the fourth limit to the last step because it is the hardest one. We prove the fifth limit first. Applying the first inequality in Lemma 4.2 with $s^{\prime}=0$ and the Hölder inequality, we have

$$
\begin{aligned}
\left|\int_{0}^{t}\left(\overline{\mathcal{D}} U_{1 \epsilon} \mid U_{2 \epsilon}\right)_{\mathbb{H}}-\left(\overline{\mathcal{D}} U_{1} \mid U_{2}\right)_{\mathbb{H}} \mathrm{d} t^{\prime}\right|= & \left|\int_{0}^{t}\left(\overline{\mathcal{D}} U_{1 \epsilon}-\overline{\mathcal{D}} U_{1} \mid U_{2 \epsilon}\right)_{\mathbb{H}}+\left(\overline{\mathcal{D}} U_{1} \mid U_{2 \epsilon}-U_{2}\right)_{\mathbb{H}} \mathrm{d} t^{\prime}\right| \\
\leq & C_{\mathcal{D}}\left\|\nabla_{x}\left(U_{1 \epsilon}-U_{1}\right)\right\|_{L^{2}([0, T] ; \mathbb{H})}\left\|\nabla_{x} U_{2 \epsilon}\right\|_{L^{2}([0, T] ; \mathbb{H})} \\
& +C_{\mathcal{D}}\left\|\nabla_{x} U_{1}\right\|_{L^{2}([0, T] ; \mathbb{H})}\left\|\nabla_{x}\left(U_{2 \epsilon}-U_{2}\right)\right\|_{L^{2}([0, T] ; \mathbb{H})} .
\end{aligned}
$$

Note that $\nabla_{x} U_{i \epsilon}=\left(\nabla_{x} U_{i}\right)_{\epsilon}$, for $i=1,2$, the boundedness of $\nabla_{x} U_{i \epsilon}$ in $L^{2}([0, T] ; \mathbb{H})$, and the convergence

$$
\left(\nabla_{x} U_{i}\right)_{\epsilon} \rightarrow \nabla_{x} U_{i} \quad \text { in } \quad L^{2}([0, T] ; \mathbb{H}) \quad \text { as } \quad \epsilon \rightarrow 0,
$$

we finish the proof of the fifth limit.

The main difficulty is that $U_{2}$ is only a Leray weak solution, so $\overline{\mathcal{Q}}\left(U_{2}, U_{2}\right)$ is not in $L^{2}$, thus the method to prove the third limit is not applicable here. However, we have the following identity: for any functions $U, V$ so that $U^{T} G_{o} V \in L^{1}\left([0, T] ; L^{1}(\mathrm{~d} x)\right)$ and $T>0$,

$$
\int_{0}^{T}\left(\Theta_{\epsilon} * U \mid V\right)_{\mathbb{H}} \mathrm{d} t=\int_{0}^{T}\left(U \mid \Theta_{\epsilon} * V\right)_{\mathbb{H}} \mathrm{d} t .
$$


Proof of (6.20): By changing the order of integration,

$$
\begin{aligned}
& \int_{0}^{T}\left(\Theta_{\epsilon} * U \mid V\right)_{\mathbb{H}} \mathrm{d} t \\
& =\int_{0}^{T} \int_{\mathbb{T}^{d}} \int_{0}^{\infty} \int_{\mathbb{T}^{d}} \frac{1}{\epsilon^{d+1}} \alpha\left(\frac{x-x^{\prime}}{\epsilon}\right) \beta\left(\frac{t-t^{\prime}}{\epsilon}\right) U^{T}\left(x^{\prime}, t^{\prime}\right) \mathrm{d} x^{\prime} \mathrm{d} t^{\prime} G_{O} V(x, t) \mathrm{d} x \mathrm{~d} t \\
& =\int_{0}^{T} \int_{\mathbb{T}^{d}} \int_{t^{\prime}}^{T} \int_{\mathbb{T}^{d}} \frac{1}{\epsilon^{d+1}} \alpha\left(\frac{x-x^{\prime}}{\epsilon}\right) \beta\left(\frac{t-t^{\prime}}{\epsilon}\right) V^{T}(x, t) \mathrm{d} x \mathrm{~d} t G_{o} U\left(x^{\prime}, t^{\prime}\right) \mathrm{d} x^{\prime} \mathrm{d} t^{\prime} \\
& =\int_{0}^{T}\left(U \mid \Theta_{\epsilon} * V\right)_{\mathbb{H}} \mathrm{d} t .
\end{aligned}
$$

Applying (6.20) to $U_{1}$ and $\overline{\mathcal{Q}}\left(U_{2}, U_{2}\right)$, we have

$$
\int_{0}^{t}\left(U_{1 \epsilon} \mid \Theta_{\epsilon} * \overline{\mathcal{Q}}\left(U_{2}, U_{2}\right)\right)_{\mathbb{H}} \mathrm{d} t^{\prime}=\int_{0}^{t}\left(\Theta_{\epsilon} * U_{1 \epsilon} \mid \overline{\mathcal{Q}}\left(U_{2}, U_{2}\right)\right)_{\mathbb{H}} \mathrm{d} t^{\prime}
$$

Thus from the second inequality in Lemma 4.2,

$$
\begin{aligned}
& \left|\int_{0}^{t}\left(\Theta_{\epsilon} * U_{1 \epsilon}-U_{1} \mid \overline{\mathcal{Q}}\left(U_{2}, U_{2}\right)\right)_{\mathbb{H}} \mathrm{d} t^{\prime}\right| \\
& \leq C_{\mathcal{Q}}^{s} T^{\frac{1}{2}} \int_{0}^{t}\left\|\nabla_{x}\left(\Theta_{\epsilon} * U_{1 \epsilon}-U_{1}\right)\right\|_{L^{\infty}\left([0, T] ; \mathbb{H}^{s}\right)} \mathrm{d} t^{\prime}\left\|U_{2}\right\|_{L^{\infty}(\mathrm{d} t ; \mathbb{H})}^{2} .
\end{aligned}
$$

Note that

$$
\nabla_{x}\left(\Theta_{\epsilon} * U_{\epsilon}\right)=\Theta_{\epsilon} *\left(\nabla_{x} U\right)_{\epsilon}
$$

We claim that for any $W \in L^{2}\left([0, T] ; \mathbb{H}^{s}\right)$,

$$
\lim _{\epsilon \rightarrow 0}\left\|\Theta_{\epsilon} * W_{\epsilon}-W\right\|_{L^{2}\left([0, T] ; \mathbb{H}^{s}\right)} \rightarrow 0 \quad \text { as } \epsilon \rightarrow 0,
$$

which simply followed from the triangle inequality

$$
\left\|\Theta_{\epsilon} * W_{\epsilon}-W\right\|_{L^{2}\left([0, T] ; \mathbb{H}^{s}\right)} \leq\left\|\Theta_{\epsilon} * W_{\epsilon}-W_{\epsilon}\right\|_{L^{2}\left([0, T] ; \mathbb{H}^{s}\right)}+\left\|W_{\epsilon}-W\right\|_{L^{2}\left([0, T] ; \mathbb{H}^{s}\right)},
$$

and the convergence property of convolution in $L^{2}\left([0, T] ; \mathbb{H}^{s}\right)$. We thereby prove the limit.

We have now established all the limits asserted in (6.18) and have thereby completed the proof of Lemma 6.1 .

\section{Application to the Compressible Navier-Stokes System}

7.1. Compressible Navier-Stokes System. The compressible Navier-Stokes system of gas dynamics is an important example of a nonsingular hyperbolic-parabolic system with the strictly convex entropy. It governs the mass density $\rho(x, t)$, bulk velocity $u(x, t)$, and temperature $\theta(x, t)$ over $\Omega \subset \mathbb{R}^{d}$ in the form

$$
\begin{aligned}
\partial_{t} \rho+\nabla_{x} \cdot(\rho u) & =0, \\
\partial_{t}(\rho u)+\nabla_{x} \cdot(\rho u \otimes u+p I+S) & =0, \\
\partial_{t}\left(\frac{1}{2} \rho|u|^{2}+\rho \varepsilon\right)+\nabla_{x} \cdot\left(\frac{1}{2} \rho|u|^{2} u+\rho \varepsilon u+p u+S \cdot u+q\right) & =0,
\end{aligned}
$$

where the specific energy $\varepsilon$ and pressure $p$ are given by thermodynamic equations-of-state, $\varepsilon=\varepsilon(\rho, \theta)$ and $p=p(\rho, \theta)$, while the stress $S$ and heat flux $q$ are given by the constitutive relations

$$
S=-\mu\left(\nabla_{x} u+\left(\nabla_{x} u\right)^{T}-\frac{2}{\mathrm{D}} I \nabla_{x} \cdot u\right)-\lambda I \nabla_{x} \cdot u, \quad q=-\kappa \nabla_{x} \theta
$$


Here the coefficients of shear viscosity $\mu$, bulk viscosity $\lambda$ and thermal conductivity $\kappa$ are given by formulas $\mu=\mu(\rho, \theta), \lambda=\lambda(\rho, \theta)$, and $\kappa=\kappa(\rho, \theta)$ that come either from a nonequilibrium (kinetic) theory or from fits to experimental data, while $\mathrm{D}$ is the dimension of the underlying microscopic world - usually $\mathrm{D}=3$. We require that $\mathrm{D} \geq \max \{2, d\}$.

Equations (7.1) express the local conservation of mass, momentum, and energy. The constitutive relations (7.2) for $S$ and $q$ model viscosity and thermal conductivity, which arise due to deviations of the gas from local thermodynamic equilibrium. Equations (7.1) reduce to the compressible Euler system when one sets $S=0$ and $q=0$.

The thermodynamic equations-of-state for the specific energy and pressure, $\varepsilon=\varepsilon(\rho, \theta)$ and $p=p(\rho, \theta)$, are assumed to be twice continuously differentiable over $(\rho, \theta) \in \mathbb{R}_{+}^{2}$ and to satisfy

$$
\partial_{\theta} \varepsilon(\rho, \theta)>0, \quad \partial_{\rho} p(\rho, \theta)>0, \quad \text { for every } \rho>0 \text { and } \theta>0 .
$$

In addition, they must satisfy the Maxwell relation

$$
\rho^{2} \partial_{\rho} \varepsilon+\theta^{2} \partial_{\theta}\left(\frac{p}{\theta}\right)=0 .
$$

This implies the existence of a function $\sigma=\sigma(\rho, \theta)$ that satisfies the differential relation

$$
\mathrm{d}\left(\sigma-\frac{\varepsilon}{\theta}\right)=-\varepsilon \mathrm{d}\left(\frac{1}{\theta}\right)+\frac{p}{\theta} \mathrm{d}\left(\frac{1}{\rho}\right) .
$$

This is equivalent to

$$
\mathrm{d} \sigma=\frac{1}{\theta} \mathrm{d} \varepsilon-\frac{p}{\rho^{2} \theta} \mathrm{d} \rho .
$$

We can identify $\sigma$ with the specific entropy.

We make use of the convective form of system (7.1),

$$
\begin{array}{r}
\partial_{t} \rho+u \cdot \nabla_{x} \rho+\rho \nabla_{x} \cdot u=0, \\
\rho\left(\partial_{t} u+u \cdot \nabla_{x} u\right)+\nabla_{x} p+\nabla_{x} \cdot S=0, \\
\rho\left(\partial_{t} \varepsilon+u \cdot \nabla_{x} \varepsilon\right)+p \nabla_{x} \cdot u+S: \nabla_{x} u+\nabla_{x} \cdot q=0,
\end{array}
$$

and the differential specific entropy relation (7.4) to see that

$$
\rho\left(\partial_{t} \sigma+u \cdot \nabla_{x} \sigma\right)=\frac{\rho}{\theta}\left(\partial_{t} \varepsilon+u \cdot \nabla_{x} \varepsilon\right)-\frac{p}{\rho \theta}\left(\partial_{t} \rho+u \cdot \nabla_{x} \rho\right)=-\frac{1}{\theta} S: \nabla_{x} u-\frac{1}{\theta} \nabla_{x} \cdot q .
$$

This can be put into the divergence form

$$
\partial_{t}(\rho \sigma)+\nabla_{x} \cdot\left(\rho u \sigma+\frac{q}{\theta}\right)=-\frac{1}{\theta} S: \nabla_{x} u-\frac{1}{\theta^{2}} q \cdot \nabla_{x} \theta .
$$

The local form of the second law of thermodynamics and the constitutive relations (7.2) imply that for any values of $\rho, \theta, \nabla_{x} u+\left(\nabla_{x} u\right)^{T}-\frac{2}{\mathrm{D}} I \nabla_{x} \cdot u, \nabla_{x} \cdot u$, and $\nabla_{x} \theta$ one has the inequality

$$
-\frac{1}{\theta} S: \nabla_{x} u-\frac{1}{\theta^{2}} q \cdot \nabla_{x} \theta=\frac{\mu}{2}\left|\nabla_{x} u+\left(\nabla_{x} u\right)^{T}-\frac{2}{\mathrm{D}} I \nabla_{x} \cdot u\right|^{2}+\lambda\left|\nabla_{x} \cdot u\right|^{2}+\kappa\left|\nabla_{x} \theta\right|^{2} \geq 0 .
$$

Because these values can be independently specified at any point in $\Omega$, the above inequality implies that $\mu(\rho, \theta) \geq 0, \lambda(\rho, \theta) \geq 0$, and $\kappa(\rho, \theta) \geq 0$ for every $\rho>0$ and $\theta>0$. Of course, 
these thermodynamic constraints are satisfied when $\mu=\lambda=\kappa=0$, which is the case of the compressible Euler system. For the compressible Navier-Stokes system we require that

$$
\mu(\rho, \theta)>0, \quad \lambda(\rho, \theta) \geq 0, \quad \kappa(\rho, \theta)>0, \quad \text { for every } \rho>0 \text { and } \theta>0,
$$

which are also consistent with the thermodynamic constraints.

Now consider the system over a periodic box $\Omega=\mathbb{T}^{d}$. Integrating the divergence form of the entropy equation (7.6) over $\mathbb{T}^{d}$ yields

$$
\frac{\mathrm{d}}{\mathrm{d} t} \int_{\mathbb{T}^{d}} \rho \sigma \mathrm{d} x=\int_{\mathbb{T}^{d}}\left[\frac{\mu}{2}\left|\nabla_{x} u+\left(\nabla_{x} u\right)^{T}-\frac{2}{\mathrm{D}} I \nabla_{x} \cdot u\right|^{2}+\lambda\left|\nabla_{x} \cdot u\right|^{2}+\kappa\left|\nabla_{x} \theta\right|^{2}\right] \mathrm{d} x .
$$

One sees from (7.7) and (7.8) that any stationary classical solution of the system for which $\rho>0$ and $\theta>0$ must satisfy

$$
\nabla_{x} u+\left(\nabla_{x} u\right)^{T}-\frac{2}{\mathrm{D}} I \nabla_{x} \cdot u=0, \quad \nabla_{x} \theta=0 .
$$

Because $u$ is periodic, one can use the first equation above to argue that $\nabla_{x} u=0$. It then follows from (7.2) and (7.5) that $\nabla_{x} p(\rho, \theta)=0$, which by the second equation above and (7.3) yields $\nabla_{x} \rho=0$. This shows that the compressible Navier-Stokes system (7.1-7.2) satisfies the nonsingularity condition (2.10).

The compressible Navier-Stokes system (7.1-7.2) is a nonsingular hyperbolic-parabolic system with a strictly convex entropy given by $H(U)=-\rho \sigma(\rho, \theta)$ where $U$ is related to $\rho$, $u$, and $\theta$ by

$$
U=\left(\begin{array}{lllll}
\rho & \rho u_{1} & \cdots & \rho u_{\mathrm{D}} & \frac{1}{2} \rho|u|^{2}+\rho \varepsilon(\rho, \theta)
\end{array}\right)^{T} .
$$

The set $\mathcal{U}$ is the range of this mapping restricted to the domain $\rho>0, u \in \mathbb{R}^{d}$, and $\theta>0$. Whenever $\theta \mapsto \varepsilon(\rho, \theta)$ is a strictly increasing function from $\mathbb{R}_{+}$onto $\mathbb{R}_{+}$then $\mathcal{U}$ is given by

$$
\left.\mathcal{U}=\left\{\begin{array}{lllll}
U_{0} & U_{1} & \cdots & U_{\mathrm{D}} & U_{\mathrm{D}+1}
\end{array}\right)^{T} \in \mathbb{R}^{\mathrm{D}+2}: U_{0}>0,2 U_{0} U_{\mathrm{D}+1}>U_{1}^{2}+\cdots+U_{\mathrm{D}}^{2}\right\} .
$$

The function $H: \mathcal{U} \rightarrow \mathbb{R}$ will be strictly convex if and only if $\partial_{\theta} \varepsilon(\rho, \theta)>0$ and the sound speed is defined [17]. These conditions are satisfied by all thermodynamic equations-of-state that satisfy $(7.3)$.

Finally, it is easily checked that the Navier-Stokes system (7.1-7.2) satisfies our Kawashimatype criterion (5.1), whereby it also satisfies the Kawashima condition (3.7). Moreover, because its weakly nonlinear-dissipative approximation will be strictly dissipative, our theory of global weak solutions applies to it.

7.2. Weakly Compressible Navier-Stokes System. A fluid dynamical system that formally includes both the acoustic and the Stokes systems is the so-called weakly compressible Stokes system

$$
\begin{aligned}
\partial_{t} \tilde{\rho}+\rho_{o} \nabla_{x} \cdot \tilde{u} & =0, \\
\rho_{o} \partial_{t} \tilde{u}+\nabla_{x}\left(\left(\partial_{\rho} p\right)_{o} \tilde{\rho}+\left(\partial_{\theta} p\right)_{o} \tilde{\theta}\right) & =\mu_{o} \nabla_{x} \cdot\left[\nabla_{x} \tilde{u}+\left(\nabla_{x} \tilde{u}\right)^{T}-\frac{2}{\mathrm{D}} \nabla_{x} \cdot \tilde{u} I\right]+\lambda_{o} \nabla_{x}\left(\nabla_{x} \cdot \tilde{u}\right), \\
\rho_{o} C_{o}^{V} \partial_{t} \tilde{\theta}+\theta_{o}\left(\partial_{\theta} p\right)_{o} \nabla_{x} \cdot \tilde{u} & =\kappa_{o} \Delta_{x} \tilde{\theta},
\end{aligned}
$$

where $C_{o}^{V}=\left(\partial_{\theta} \varepsilon\right)_{o}$ is the specific heat capacity at constant volume. A so-called weakly compressible Navier-Stokes system that formally includes both the acoustic and the Navier-Stokes systems is the weakly nonlinear-dissipative approximation. It decomposes $\widetilde{U}$ into a component governed by the incompressible Navier-Stokes system and a component governed by a nonlocal quadratic acoustic equation that couples to the incompressible component. 
The incompressible component $\widetilde{U}_{i n}=(\tilde{\rho}, \tilde{u}, \tilde{\theta})$ is governed by

$$
\begin{aligned}
\rho_{o}\left(\partial_{t} \tilde{u}+\tilde{u} \cdot \nabla_{x} \tilde{u}\right)+\nabla_{x} \tilde{p} & =\mu_{o} \Delta_{x} \tilde{u}, \\
\rho_{o} C_{o}^{P}\left(\partial_{t} \tilde{\theta}+\tilde{u} \cdot \nabla_{x} \tilde{\theta}\right) & =\kappa_{o} \Delta_{x} \tilde{\theta},
\end{aligned}
$$

where

$$
\nabla_{x} \cdot \tilde{u}=0, \quad\left(\partial_{\rho} p\right)_{o} \tilde{\rho}+\left(\partial_{\theta} p\right)_{o} \tilde{\theta}=0,
$$

and $C_{o}^{P}$ denotes the specific heat capacity at constant pressure, while the acoustic component $\widetilde{U}_{a c}=(\tilde{\eta}, \tilde{v}, \tilde{\chi})$ is governed by

$$
\partial_{t} \widetilde{U}_{a c}+\mathcal{A} \widetilde{U}_{a c}+\nabla_{x} \cdot \bar{Q}_{i n}\left(\widetilde{U}_{i n}, \widetilde{U}_{a c}\right)+\nabla_{x} \cdot \bar{Q}_{a c}\left(\widetilde{U}_{a c}, \widetilde{U}_{a c}\right)=\bar{\nu} \Delta_{x} \widetilde{U}_{a c}
$$

where

$$
\nabla_{x} \wedge \tilde{v}=0, \quad \tilde{\chi}=\frac{\theta_{o}\left(\partial_{\theta} p\right)_{o}}{\rho_{o}^{2} C_{o}^{V}} \tilde{\eta},
$$

The diffusion coefficient $\bar{\nu}$ in (7.12) is given by

$$
\bar{\nu}=\frac{2 \frac{\mathrm{D}-1}{\mathrm{D}} \mu_{o}+\lambda_{o}}{2 \rho_{o}}+\frac{\kappa_{o}}{2 \rho_{o} C_{o}^{V}} \frac{\theta_{o}\left(\partial_{\theta} p\right)_{o}^{2}}{\rho_{o}^{2} C_{o}^{V} c_{o}^{2}}
$$

where $c_{o}$ is the sound speed defined by

$$
c_{o}^{2}=\left(\partial_{\rho} p\right)_{o}+\frac{\theta_{o}\left(\partial_{\theta} p\right)_{o}^{2}}{\rho_{o}^{2} C_{o}^{V}} .
$$

Notice that $\bar{\nu}$ is positive if either $\mu_{o}, \lambda_{o}$, or $\kappa_{o}$ is positive! $\bar{Q}_{i n}$ and $\bar{Q}_{a c}$ are nonlocal operators defined in the following way. We first introduce the orthonomal basis of the acoustic mode $\operatorname{Null}(\mathcal{A})^{\perp}$ :

$$
H_{k}^{ \pm}(x)=\sqrt{\frac{\theta_{o}}{2 \rho_{o}}}\left(\begin{array}{c}
\frac{\rho_{o}}{c_{o}} \\
\pm \frac{k}{|k|} \\
\frac{\theta_{o}\left(\partial_{\theta} p\right)_{o}}{\rho_{o} C_{o}^{V} c_{o}}
\end{array}\right) e^{i k \cdot x}
$$

It is easy to check that $H_{k}^{ \pm}$is the eigenvector of the acoustic operator $\mathcal{A}$ with eigenvalues $\pm i c_{o}|k|$. Then any acoustic component $\widetilde{U}_{a c}$ can be represented as

$$
\widetilde{U}_{a c}=\sum_{k} U_{k}^{ \pm} H_{k}^{ \pm}(x)
$$

where $U_{k}^{ \pm}$is the coefficient of $\widetilde{U}_{a c}$ with respect to the basis $H_{k}^{ \pm}$under the inner product $(\cdot \mid \cdot)_{\mathbb{H}}$. The nonlocal operator $\bar{Q}_{i n}$ can be written as

$$
\bar{Q}_{i n}\left(\widetilde{U}_{i n}, \widetilde{U}_{a c}\right)=\sum_{\delta, m} \lambda_{m}^{ \pm}\left(\widetilde{U}_{i n}\right) H_{m}^{ \pm}(x),
$$

where

$$
\lambda_{m}^{ \pm}\left(\widetilde{U}_{i n}\right)=\sum_{\substack{ \pm k \pm l=m \\|k|=|m|}} U_{k}^{ \pm}\left[c_{1} \frac{\left(\widehat{\tilde{u}}_{l} \cdot m\right) k+(k \cdot m) \widehat{\tilde{u}}_{l}}{|k \| m|}+\frac{\widehat{\tilde{\theta}}_{l}}{|m|}\left(c_{2} k+c_{3} m\right)\right]
$$


The nonlocal operator $\bar{Q}_{a c}$ can be written as

$$
\bar{Q}_{i n}\left(\widetilde{U}_{a c}, \widetilde{U}_{a c}\right)=\sum_{m} \chi_{m}^{ \pm} H_{m}^{ \pm}(x),
$$

where

$$
\chi_{m}^{ \pm}=c_{4} \sum_{\substack{k+l=m \\ \pm(k)|k|+ \pm(l)|l|= \pm|m|}} U_{k}^{ \pm} U_{l}^{ \pm} \frac{m}{|m|},
$$

where the constants $c_{1}, c_{2}, c_{3}$, and $c_{4}$ are calculated in [22].

We have shown that the weakly compressible Navier-Stokes system has global weak solutions in $L^{2}$. This result includes the Leray theory, so it cannot be improved easily. As with the Leray theory, the key to this result is an "energy" dissipation estimate. We have, (extending ideas of Masmoudi and Danchin) also adapted a Littlewood-Payly decomposition to show the acoustic part is unique for a given incompressible component [21]. Moreover, we have derived the weakly compressible Navier-Stokes system directly from the Boltzmann equation for the case $p=\rho \theta$ and $\varepsilon=\frac{\mathrm{D}}{2} \theta[22]$.

\section{REFERENCES}

[1] A. Babin, A. Mahalov, and B. Nicolaenko, Global regularity and integrability of $3 D$ Euler and Navier-Stokes Equations for Uniformly Rotating Fluids, Asymptotic Analysis 15 (1997), 103-150.

[2] A. Babin, A. Mahalov, and B. Nicolaenko, Global Regularity of 3D Rotating Navier-Stokes Equations for Resonant Domains, Indiana Univ. Math. J. 48, (1999) 1133-1176.

[3] A. Babin, A. Mahalov, and B. Nicolaenko, 3D Navier-Stokes and Euler Equations with Initial Data Characterized by Uniformly Large Vorticity, Indiana Univ. Math. J. 50 (2001) 1-35.

[4] S. Bianchini, B. Hanouzet, and R. Natalini, Asymptotic behavior of smooth solutions for partially dissipative hyperbolic systems with a convex entropy, Commun. on Pure \& Appl. Math. 60 (2007), no. 11, 1559-1622

[5] G.-Q. Chen, C.D. Levermore, and T.-P. Liu, Hyperbolic Conservation Laws with Stiff Relaxation Terms and Entropy, Commun. Pure \& Appl. Math. 47 (1994), 787-830.

[6] P. Constantin and C. Foias, Navier-Stokes Equations, Chicago Lectures in Mathematics, The University of Chicago Press, Chicago, 1988.

[7] C. Dafermos, Hyperbolic Conservation Laws in Continuum Physics, 2nd Edition, Springer-Verlag, BerlinHeidelberg, 2005.

[8] R. Danchin, Zero Mach Number Limit for Compressible Flows with Periodic Boundary Conditions, Amer. J. Math. 124:6 (2002), 1153-1219.

[9] L. Desvillettes, Hypocoercivity: The Example of Linear Transport, in Recent Trends in Partial Differential Equations, X. Cabré, J.A. Carrillo, and J.L. Vázquez eds., Contemp. Math. 409, 33-54, Amer. Math. Soc., Providence, RI, 2006

[10] C.R. Doering, J.D. Gibbon, and C.D. Levermore, Weak and Strong solutions of the Complex GinzburgLandau Equation, Physica D 71 (1994), 285-318.

[11] P. Embid and A.J. Majda, Averaging over Fast Gravity Waves for Geophyical Flows with Arbitrary Potenital Vorticity, Commun. P.D.E. 21 (1996), 619-658.

[12] P. Embid and A.J. Majda, Low Froude Number Limiting Dynamics for Stably Stratified Flow with Small or Finite Rossby Numbers, Geophys.\& Astrophys. Fluid Dynamics 87 (1998), 1-50.

[13] K.O. Friedrichs and P.D. Lax, Systems of Conservation Equations with a Convex Extension, Proc. Nat. Acad. Sci. USA 68 (1971), 1686-1688.

[14] I. Gallagher Asymptotics of the solutions of hyperbolic equations with a skew-symmetric perturbation, Journal of Differential Equations, 150 (1998), 363-384.

[15] I. Gallagher Applications of Schochet's Methods to Parabolic Equations, Journal de Mathmatiques Pures et Appliques, 77 (1998), 989-1054.

[16] S. K. Godunov, An Interesting Class of Quasilinear Systems, Dokl. Acad. Nauk SSSR 139 (1961), 521-523. 
[17] A. Harten, P.D. Lax, C.D. Levermore, and W.J. Morokoff, Convex Entropies and Hyperbolicity for General Euler Equations, SIAM J. Num. Anal. 35 (1998), 2117-2127.

[18] D. Hoff and K. Zumbrun, Multi-Dimensional Diffusion Waves for the Navier-Stokes Equations of Compressible Flow, Indiana Univ. Math. J. 44:2 (1995), 603-676.

[19] D. Hoff and K. Zumbrun, Pointwise Decay Estimates for Multidimensional Navier-Stokes Diffusion Waves, Z. Angew. Math. Phys. 48:4 (1997), 597-614.

[20] L. Hörmander, Hypoelliptic second order differential operators, Acta Math. 119 (1967), 147-171.

[21] N. Jiang and C.D. Levermore, Global Existence and Regularity of Weakly Compressible Gas Dynamics, Preprint 2009.

[22] N. Jiang and C.D. Levermore, Weakly Compressible Stokes Dynamics of the Boltzmann Equation, Preprint 2009.

[23] S. Kawashima, Systems of Hyperbolic-Parabolic Composite Type, with Application to the Equations of Magnetohydrodynamics, Doctoral Thesis, Kyoto University, 1984.

[24] Y. Shiazuta and S. Kawashima, System of equations of hyperbolic-parabolic type with applications to the discrete Boltzmann equation, Tohoku Math. J. 14 (1985), 249-275.

[25] S. Kawashima, Large-time behaviour of solutions to hyperbolic-parabolic systems of conservation laws and applications, Proc. Roy. Soc. Edinburgh Sect. A 106 (1987), no. 1-2, 169-194.

[26] S. Kawashima, Large-time behavior of solutions of the discrete Boltzmann equation, Comm. Math. Phys. 109 (1987), no. 4, 563-589.

[27] O.A. Ladyzhenskaya, The Mathematical Theory of Viscous Incompressible Flow, Gordon and Breach, New York, 1969

[28] J. Leray, Sur le mouvement d'un fluide visqueux emplissant l'espace, Acta Math. 63 (1934), 193-248.

[29] P.L. Lions and N. Masmoudi, Incompressible limit for a viscous compressible fluid, J. Math. Pures Appli. 77 (1998), 585-627.

[30] P. L. Lions and N. Masmoudi, Une approche locale de la limite incompressible, C. R. Acad. Sci. Paris Sr. I Math. 329 (1999), 387-392.

[31] T.-P. Liu and Y. Zeng, Large Time Behavior of Solutions for General Quasilinear Hyperbolic-Parabolic Systems of Conservation Laws, Mem. Amer. Math. Soc. 125 (1997), 599.

[32] N. Masmoudi, Incompressible, Inviscid Limit of the Compressible Navier-Stokes System, Ann. Inst. H. Poincaré 18 (2001), 199-224.

[33] M. Puel and L. Saint-Raymond, Quasineutral Limit for the Relativistic Vlasov-Maxwell System, Asymptotic Analysis 40 (2004), 303-352.

[34] T. Ruggeri and D. Serre, Stability of constant equilibrium state for dissipative balance laws system with a convex entropy, Quart. Appl. Math. 62 (2004), no. 1, 163-179.

[35] S. Schochet, Fast Singular Limits of Hyperbolic PDE's, J. Diff. Equations 114 (1994), 476-512.

[36] R. Temam, Navier-Stokes Equations: Theory and Numerical Analysis. AMS Chelsea, Providence, 2001.

[37] W-A, Yong, Entropy and global existence for hyperbolic balance laws. Arch. Ration. Mech. Anal. 172 (2004), no. 2, 247-266.

[38] C. Villani, Hypocoercivity, Memoirs Amer. Math. Soc. (to appear).

Department of Mathematics \& Center for Scientific Computation and Mathematical Modeling (CSCAMM), University of Maryland, College Park, MD 20742

Current address: Courant Institute of Mathematical Sciences, 251 Mercer Street, New York, NY 10012, USA

E-mail address: njiang@cims.nyu.edu

Department of Mathematics \& Institute for Physical Science and Technology (IPST), University of Maryland, College Park, MD 20742-4015, USA

E-mail address: lvrmr@math.umd.edu 